\title{
A study on relativistic lagrangian field theories with non-topological soliton solutions
}

\author{
J. Diaz-Alonso ${ }^{\mathrm{a}, \mathrm{b}, *}$, D. Rubiera-Garcia ${ }^{\mathrm{b}}$ \\ ${ }^{a}$ LUTH, Observatoire de Paris, CNRS, Université Paris Diderot. 5 Place Jules Janssen, 92190 Meudon, France \\ ${ }^{\mathrm{b}}$ Departamento de Física, Universidad de Oviedo. Avda. Calvo Sotelo 18, E-33007 Oviedo, Asturias, Spain
}

\begin{abstract}
We perform a general analysis of the dynamic structure of two classes of relativistic lagrangian field theories exhibiting static spherically symmetric non-topological soliton solutions. The analysis is concerned with (multi-) scalar fields and generalized gauge fields of compact semi-simple Lie groups. The lagrangian densities governing the dynamics of the (multi-) scalar fields are assumed to be general functions of the kinetic terms, whereas the gaugeinvariant lagrangians are general functions of the field invariants. These functions are constrained by requirements of regularity, positivity of the energy and vanishing of the vacuum energy, defining what we call "admissible" models. In the scalar case we establish the general conditions which determine exhaustively the families of admissible lagrangian models supporting this kind of finite-energy solutions. We analyze some explicit examples of these different families, which are defined by the asymptotic and central behaviour of the fields of the corresponding particle-like solutions. From the variational analysis of the energy functional, we show that the admissibility constraints and the finiteness of the energy of the scalar solitons are necessary and sufficient conditions for their linear static stability against small charge-preserving perturbations. Furthermore we perform a general spectral analysis of the dynamic evolution of the small perturbations around the statically stable solitons, establishing their dynamic stability. Next, we consider the case of many-components scalar fields, showing that the resolution of the particle-like field problem in this case reduces to that of the one-component case. The study of these scalar models is a necessary step in the analysis of the gauge fields. In this latter case, we add the requirement of parity invariance to the admissibility constraints. We determine the general conditions defining the families of admissible gauge-invariant models exhibiting finiteenergy electrostatic spherically symmetric solutions which, unlike the (multi-) scalar case, are not always stable. The variational analysis of the energy functional leads now to supplementary restrictions to be imposed on the lagrangian densities in order to ensure the linear stability of the solitons. We establish a correspondence between any admissible soliton-supporting (multi-) scalar model and a family of admissible generalized gauge models supporting finite-energy electrostatic point-like solutions. Conversely, for each admissible soliton-supporting gauge-invariant model there is an associated unique admissible (multi-) scalar model with soliton solutions. This shows the exhaustive character of the admissibility and stability conditions in determining the class of soliton-supporting generalized gauge models. The usual Born-Infeld electrodynamic theory and its non-abelian extensions are shown to be (very particular) examples of one of these families.
\end{abstract}

Key words: non-linear field theories, solitons, gauge field theories, stability, Born-Infeld model PACS: 05.45.Yv, 11.10.-z, 11.10.Lm, 11.15.-q 


\section{Introduction}

In the context of field theory the interest in extended configurations describing fields associated to point-like particles (particle-like solutions) dates back to the works of Mie [1] and Born and Infeld (B-I) [2],[3], aimed to solve the problem of the divergent self-energy of the electron field in Classical Electrodynamics. Today, this kind of problems fall inside the large domain of Soliton Physics, whose methods and applications concern most branches of physical sciences.

Although there is not an universally accepted definition of the concept of soliton, we shall adopt here minimal defining properties which seem to be widely assumed in most contexts: Solitons are stable, finite-energy solutions of conservative non-linear differential equations. However, the accepted meaning and content of the term stable is not universal. In a strong sense, it refers to the existence of soliton entities which can be identified (if present) in field configurations and are preserved by the dynamic evolution of the system. With this definition, the analysis of such configurations in terms of many solitons, interacting via radiative field exchanges, becomes possible. This kind of stability arises in some field theoretical models (most of them in one-space dimension) exhibiting topological conservation laws, related to a non-trivial structure of the vacuum [4]. In these cases the conserved topological charges identify the presence of the topological solitons and ensure their preservation. Examples of topological solitons in three-space dimensions are the monopole of 't Hooft and Polyakov [5] or the Skyrmion [6]. In a weak sense, stability is identified with linear stability, i.e. with the preservation of the soliton identity against a certain class of small perturbations for which the soliton configuration is a minimum of the functional of energy. This restricted class of perturbations is defined through boundary conditions which amount, in general, to the preservation of (non-topological) charges associated with the soliton. The conservation laws of these charges may be implicitly contained within the structure of the field equations (as in electrodynamics) or be consequences of constraints imposed on the external sources, to which the field is coupled. Here we shall be only concerned with this kind of non-topological, finite-energy, weakly-stable soliton solutions of local relativistic lagrangian field theories in three-space dimensions.

Non-linear field theories supporting soliton solutions have been widely studied and applied in several contexts of Theoretical Physics. Let us give some examples:

1) In the context of elementary particle physics, let us mention the phenomenological description of the hadron structure and the hadronic interactions in terms of topological solitons in the framework of the Skyrme model [6]. Other descriptions of this structure in terms of non-topological solitons have been performed through the effective approach to the low-energy regime of Quantum Chromodynamics (QCD) given by the Friedberg-Lee model [7], [8] and related theories. Let us also mention the generalized chiral-invariant model of Deser, Duff and Isham [9], whose lagrangian density is a rational power $(3 / 2)$ of the nonlinear sigma model lagrangian, chosen in order to circumvent Derrick's theorem [10]. This model and its extensions support topological solitons [11. In the same context let us also

\footnotetext{
* Corresponding author.

Email address: joaquin.diaz@obspm.fr (J. Diaz-Alonso).
} 
mention the toroidal solitons of Ref. [12, which might describe glueball collective states in the low-energy limit of QCD, as suggested in Ref. [13].

2) In nuclear physics, the analysis of high-density hadronic matter and its chiral phase structure has been performed in terms of systems of skyrmions in the framework of effective field theories implemented with the large $N_{c}$ behaviour of QCD [14].

3) The glueball collective states, as soliton solutions of non-abelian B-I gauge field models [15]. The introduction of these generalized gauge models supporting soliton solutions and their extensions to higher dimensions is suggested by string theory, since some of them arise in the low-energy limit of D-Branes [16]. Moreover, it has been shown that the B-I extension of the basic lagrangian of the Skyrme model leads to stable soliton solutions. This extension was proposed as an alternative to the inclusion of the quartic "Skyrme term" in the original lagrangian of the non-linear sigma model [17]. This "ad hoc" term stabilizes the soliton by preventing Derrick's scaling, but its introduction is not justified from more fundamental reasons.

4) In the last two decades there has been an increasingly amount of works on selfgravitating field configurations. The aforementioned presence of B-I actions in the low-energy physics of D-branes, whose fundamental excitation is gravity, is one of the motivations for this renewed interest (see e.g. [18]). But the search for self-gravitating field configurations, as solutions of the Einstein equations for gravity coupled to different kinds of fields, is an older topic [19]. Indeed, in a four-dimensional flat space-time, Derrick's theorem [10] and several non-existence theorems [20] limit drastically the class of lagrangian field theories supporting soliton solutions. However, the coupling to gravity can remove these obstructions and thus allow for particle-like solutions in some cases. One example of this is the pure Yang-Mills theory, which does not support glueball solutions in Minkowski space [20] but exhibits particle-like solutions in curved space-time [21]. Another example is the regular black-hole solution of Einstein's equations coupled to a non-linear electrodynamics [22]. Let us also mention that theories supporting soliton solutions in flat space, as the abelian and non-abelian Born-Infeld models, have been extended to curved space leading to blackhole-like soliton solutions [23. Finally, we cite the soliton stars introduced by T.D. Lee as self-gravitating coherent quantum states with the features of non-topological solitons [8], [24].

5) In the fast-evolving context of modern Cosmology let us mention some problems for which non-linear field theories supporting soliton solutions have been invoked. It has been suggested that time-dependent but non-dispersive scalar solitons (Q-balls [25]) may account for the behaviour of self-interacting dark matter [26]. There is also the suggestion that nontopological solitons might have been formed in a second-order phase transition in the early Universe, and contribute significantly to the present mass density [27]. As another example, the Born-Infeld generalization of SU(2) non-abelian gauge field theory, coupled to tensorscalar gravitation, has been used for the description of dark energy [28]. Also scalar field models with lagrangian densities which are generalized functions of the kinetic term have been used to drive inflationary evolution in the early Universe (k-inflation) [29]. Solitonic configurations of these k-essence fields have been used to reproduce some properties of dark matter as well [30].

All these considerations underline the interest of non-linear lagrangian field theories and their eventual soliton solutions, mainly for the cases of (one and many-components) scalar 
field models and generalized gauge-invariant field theories. By generalized gauge field theories we mean models for gauge fields of compact semi-simple Lie groups, with lagrangian densities defined as general functions $\varphi(X, Y)$ of the two standard first-order gauge invariants, namely $X=\operatorname{tr}\left(F_{\mu \nu} \cdot F^{\mu \nu}\right)$ and $Y=\operatorname{tr}\left(F_{\mu \nu} \cdot F^{* \mu \nu}\right)$. Aside from the already mentioned Born-Infeld-like gauge models, defined by the very particular B-I choice of this function, there are not in the literature systematic studies on solitons for general gauge-invariant field theories. It is one of the main purposes of this work to perform a study on this class of theories in three space dimensions, by setting the conditions on the lagrangian functions determining the families of these models which are physically admissible and support elementary solutions which are non-topological solitons 1 . The requirements for admissibility adopted here refer to the positive-definite character of the energy, the vanishing of the vacuum energy and the regularity, uniqueness and definiteness in all space of the elementary solutions. These conditions are targeted to deal with physically meaningful theories.

In principle, generalized gauge-invariant lagrangians are candidates to describe the dynamics of the gauge fields in gauge theories of fundamental interactions. If one accepts the fundamental character of string theory and the aforementioned results, referenced in [16], the description of the gauge-field dynamics through some generalized lagrangians, regarded as effective field models of string theory, could be more "fundamental" than the usual Maxwell-like choice $\varphi(X, Y) \sim X$, a "minimal prescription" which should be understood as a low-energy (or weak-field) approximation limit. Nevertheless, from the field-theory point of view, this minimal prescription is generally assumed to describe the fundamental dynamics of the gauge fields in the gauge-invariant lagrangians with coupling to other (generally fermionic) sectors. In this case, when the high-energy degrees of freedom are integrated out in the path integral of the original action, generalized gauge-invariant models emerge as effective lagrangians, containing new non-linear self-couplings of the gauge fields which account, at a classical phenomenological level, for quantum effects and interactions with the removed heavy-mode sector [31. Historically, the first example of this kind of effective lagrangians was obtained by Heisenberg and Euler [32] in the context of Quantum Electrodynamics (QED). It accounts for the non-linear effects of the Dirac vacuum on low-energy electromagnetic wave propagation, calculated to lowest order in the fine structure constant. When higher order operators are included we are lead to a sequence of effective lagrangians which take the form of polynomials in the field invariants, arranged as an expansion in operators of increasing dimensions [33. An interesting question arises here, related to the possibility that these effective lagrangians could exhibit soliton solutions, even though the bare lagrangian does not. In the following sections we shall give explicit examples for which the soliton elementary solutions of an effective model may be interpreted as finite-energy fields of point-charges screened by the vacuum effects, whereas the elementary field of the bare theory is energy-divergent.

For scalar fields, Derrick's theorem [10] imposes severe restrictions on the lagrangian models supporting time-independent soliton solutions in three space dimensions. One of the ways to circumvent the hypothesis of Derrick's theorem is based on the choice of the lagrangian

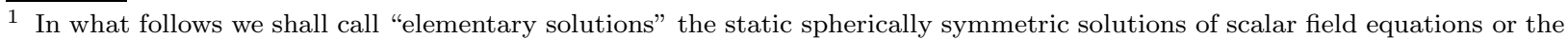
electrostatic spherically symmetric solutions of generalized gauge field equations and we shall use the acronyms SSS and ESS, respectively.
} 
density as a function of the kinetic term alone 34] (see Eq.(4.1) below). This choice seems rather arbitrary from a physical point of view. Nevertheless, it is the natural restriction for scalar fields of the lagrangian densities of the generalized gauge-invariant models. Moreover, as we shall see in the sequel, many of the necessary results in the analysis of the soliton problem in generalized (abelian and non-abelian) gauge-invariant theories (characterization of the families of soliton-supporting lagrangians, explicit determination of such models, analysis of stability etc.) can be obtained from similar results more easily established for scalar models. Consequently, the detailed study of these scalar models will take an important place in this work.

The paper is organized as follows:

In section 2 we consider the scalar field models in detail. Many general results concerning the properties of these families of scalar field theories and their associated soliton solutions can be obtained without the specification of the explicit form of the lagrangian functions. After an initial discussion on the admissibility conditions to be imposed on these functions, we solve the field equations for SSS solutions of generic models. This is achieved by obtaining the generic expression of a first-integral, which allows the determination of the field strength once the form of the lagrangian function is specified. We analyze the expression of the integral of energy for these solutions and determine the conditions that must be satisfied (at $r=0$ and as $r \rightarrow \infty$ ) in order for them to be of finite-energy. These conditions imply supplementary restrictions to be imposed on the lagrangian functions which allow for an exhaustive characterization of the admissible models supporting finite-energy SSS solutions. We shall leave for section 6 the study of the stability of the elementary solutions for these and other models.

In section 3 we introduce explicit examples of the different families of admissible scalar models supporting SSS soliton solutions. The first example is a large family of polynomial lagrangian functions including the scalar versions of the Euler-Heisenberg effective lagrangian of Electrodynamics and the sequence of higher-order effective corrections. From these examples and the results of section 5 on generalized electromagnetic field theories it can be shown that the sequence of effective lagrangians for Electrodynamics describing low-energy photon-photon interaction, obtained in the perturbative expansion [33], exhibit finite-energy point-like solutions. The second example is a two-parameter family generalizing the scalar version of the Born-Infeld model. Both the first and second examples exhibit soliton solutions which are asymptotically coulombian and differ by their behaviour at the center. The third example is a three-parameter family of models behaving like the B-I one at the center, but exhibiting diverse asymptotic behaviours. The fourth example is a three-parameter family that supports exponentially-damped soliton solutions. When properly extended to the generalized gauge-invariant case we obtain examples of gauge theories where non-linear self-couplings lead to short-ranged interactions without any symmetry breaking mechanism.

In section 4 we extend the results of the analysis of one-component scalar fields to the case of $N$-components scalar fields. For a given form of the lagrangian density as a function of the rotationally-invariant kinetic term $\left(X=\sum_{i=1}^{N} \partial_{\mu} \phi_{i} \partial^{\mu} \phi_{i}\right)$ the $N$ components of the SSS solutions have the same form, as functions of $r$, as the SSS solution of the one-component model associated to the same form of the lagrangian density function. The scalar charge of the associated one-component solution corresponds to the mean-square of the $N$ external 
scalar charges associated to the $N$-component solutions and the energy (when finite) is the same in both cases. There is thus a degeneracy of the energy on spheres of the charge space in the $N$-component case. These results will be useful in the subsequent analysis of the generalized non-abelian gauge-invariant models.

In section 5 we consider the abelian and non-abelian generalized gauge-invariant theories. After defining the dynamic problem and the admissibility conditions, we analyze the equations for ESS fields. We prove that the solutions of these equations, in the abelian and non-abelian cases, can be built from SSS solutions of the one and many-components scalar field problems, respectively. As a consequence there is a correspondence between scalar models and families of generalized gauge models in such a way that the SSS solutions and the corresponding ESS solutions have the same functional form. Moreover, if the energy of a SSS solution is finite, so is the energy of the ESS solutions of the corresponding generalized gauge family. The results of section 2 characterizing the admissible scalar models with finite-energy SSS soliton solutions, characterize also the admissible generalized gauge models with finite-energy ESS solutions, but the latter ones are not always stable. Stability requires now supplementary conditions to be satisfied by the lagrangian densities, which will be determined in section 6 .

Section 6 is devoted to a detailed analysis on the linear stability of the elementary solutions of scalar and gauge models. This analysis leads to necessary and sufficient conditions for the stability of the soliton solutions, going beyond the necessary conditions demanded by Derrick's theorem. For (multi-) scalar models the variational study of the functional of energy and the spectral analysis of the small perturbations around the SSS solutions prove the static and dynamic stability of all finite-energy SSS solutions of admissible lagrangians. For admissible abelian and non-abelian generalized gauge models, a similar analysis allows to determine supplementary (necessary and sufficient) conditions to be satisfied by the lagrangians in order for their finite-energy ESS solutions to reach stability.

We conclude in section 7 by drawing some perspectives and future developments.

Our analysis deals with fields in three-space dimensions, but most of our results can be straightforwardly generalized to other spatial dimensions.

Short reports of some of the main results developed here have been already published in Refs. [35] and [36].

\section{Scalar solitons}

We begin with lagrangian densities for scalar field potentials $\phi(x)$, defined in a four dimensional Minkowski space-time as

$$
L=f\left(\partial_{\mu} \phi \cdot \partial^{\mu} \phi\right)
$$

where $f(X)$ is a given continuous and derivable function in the domain of definition $(\Omega)$ which is assumed to be open, connected and including the vacuum $\left(X \equiv \partial_{\mu} \phi \cdot \partial^{\mu} \phi=0\right)$. For future purposes, we also require $f(X)$ to be monotonically increasing (more precisely, $d f / d X>0, \forall X \neq 0 \in \Omega$ and $d f / d X \geq 0$ for $X=0)$ and $d f / d X$ to be continuous for $X<0(X \in \Omega)$. In absence of a coupling to external sources, the associated field equations take the form of a local conservation law 


$$
\partial_{\mu} J^{\mu}=0,
$$

where the conserved current $J^{\mu}$ is

$$
J^{\mu}=\stackrel{f}{f}(X) \partial^{\mu} \phi
$$

with $f(X)=d f / d X$. In these models, the D'Alembert linear wave equation corresponds to $2 f(X)=X / 2$. For the SSS solutions $\phi(r)$, Eq.(2.2) has the first-integral

$$
r^{2} \phi^{\prime} \dot{f}\left(-\phi^{\prime 2}\right)=\Lambda,
$$

where $\phi^{\prime}=d \phi / d r$, and $\Lambda$ is the integration constant. This is an algebraic equation which allows, in principle, the determination of the field strength as a function of $r$ and $\Lambda$. The positivity of $\dot{f}(X)$ requires both $\Lambda$ and $\phi^{\prime}(r)$ to be simultaneously either positive or negative. We can then consider only the positive-sign case without loss of generality. Strictly speaking Eq.(2.4) determines the field $\phi^{\prime}(r)$ only for $r>0$. If we replace the solutions of Eq.(2.4) in Eq.(2.2) we do not obtain zero, but a Dirac $\delta$ distribution of weight $4 \pi \Lambda$. We can then identify this parameter with the central scalar charge source of the (at rest) SSS solution, in analogy with the point-like charges in the Maxwell theory. Alternatively, in some cases as, for instance, the non-linear electromagnetism of Born-Infeld, this charge may be interpreted as a continuous charge density distribution in space. For non-linear electromagnetic models the continuous interpretation of the charge is rather natural, owing to the conservation of the electric charge as a consequence of the field equations, but this is not so for the scalar models [37]. Nevertheless, following the analogy with the electromagnetic case, we can define for the models (2.1) the total scalar charge associated with a given static asymptotically vanishing field solution $\phi(\vec{r})$ as

$$
\frac{1}{4 \pi} \int d_{3} \vec{r}(\vec{\nabla} \cdot[\dot{f}(X) \vec{\nabla}(\phi)])=\frac{1}{4 \pi} \int_{S_{\infty}} \dot{f}(X) \vec{\nabla}(\phi) \cdot d \vec{\sigma},
$$

which, owing to the field equation (2.2), vanishes for everywhere-regular solutions. For the SSS solutions of (2.4) we have $\dot{f}(X) \vec{\nabla}(\phi)=\Lambda \frac{\vec{r}}{r^{3}}$ and the total charge equals $\Lambda$. We can then define the spatial charge-density distribution as $\sigma(r)=(1 / 4 \pi) \dot{f}(0) \vec{\nabla}^{2} \phi$, which gives for the total scalar charge of the SSS solutions

$$
\frac{1}{4 \pi} \int d_{3} \vec{r} \dot{f}(0) \vec{\nabla}^{2} \phi=\lim _{r \rightarrow \infty} \dot{f}(0) r^{2} \phi^{\prime}(r)=\lim _{r \rightarrow \infty} \frac{\Lambda \dot{f}(0)}{\dot{f}(X)} .
$$

Clearly, this interpretation is only possible if $\dot{f}(0)$ is finite, or equivalently, if the function $r^{2} \phi^{\prime}(r)$ goes to a constant as $r \rightarrow \infty$ (asymptotically coulombian fields). This function must also vanish as $r \rightarrow 0$. As we shall see at once, this latter condition is fulfilled for all models with finite-energy SSS solutions, but the former defines a sub-class of those models (see case B-2 below in this section) to which the scalar version of the Born-Infeld model belongs.

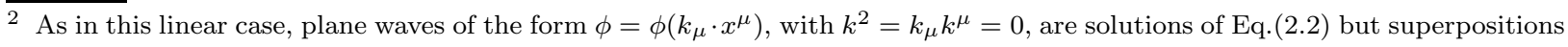
of such waves are not, in general. 
Once the form of $f(X)$ is fixed, equation (2.4) gives the expression of the field $\phi^{\prime}(r) \equiv$ $\phi^{\prime}(r, \Lambda)$ in implicit form and allows the determination of the potential $\phi(r)$ (up to an additive arbitrary constant) through a quadrature. We also note that Eq.(2.4) implies that $\phi^{\prime}(r)$, if single-branched 3 , is necessarily a monotonic function of $r$. Moreover, the solution depends on $r$ and $\Lambda$ through the ratio $r / \sqrt{\Lambda}$. This is a straightforward consequence of the invariance of the solutions of the field equations (2.2) under space-time scale transformations. Indeed, if $\phi(\vec{r}, t)$ is a solution of this equation, so is the modified function

$$
\varphi(\vec{r}, t, \lambda)=\lambda^{-1} \phi(\lambda \vec{r}, \lambda t),
$$

$\lambda$ being a positive constant. This is a symmetry of the solutions of the field equations without sources, but not an invariance of the action [38.

The canonical energy-momentum tensor associated to the lagrangian (2.1) is

$$
T_{\mu \nu}=2 \dot{f}(X) \partial_{\mu} \phi \partial_{\nu} \phi-f(X) \eta_{\mu \nu}
$$

and the energy density in the SSS case becomes

$$
\rho=T^{00}=-f\left(-\phi^{\prime 2}\right),
$$

whereas the total energy is

$$
\epsilon(\Lambda)=-4 \pi \int_{0}^{\infty} r^{2} f\left(-\phi^{\prime 2}(r, \Lambda)\right) d r=\Lambda^{3 / 2} \epsilon(\Lambda=1),
$$

the last equality being a consequence of the aforementioned scale invariance. Using the firstintegral (2.4) and integrating by parts we obtain the following useful expression for the energy in this SSS case

$$
\epsilon(\Lambda)=\frac{8 \pi \Lambda}{3}\left\{\left.[\phi(r, \Lambda)]\right|_{0} ^{\infty}-\left.\left[r \phi^{\prime}(r, \Lambda)+\frac{r^{3}}{2 \Lambda} f\left(-\phi^{\prime 2}(r, \Lambda)\right)\right]\right|_{0} ^{\infty}\right\} .
$$

As we shall see, if the energy of the SSS solutions is finite the second bracket in the r.h.s. vanishes and this expression reduces to

$$
\epsilon(\Lambda)=\frac{8 \pi \Lambda}{3}[\phi(\infty, \Lambda)-\phi(0, \Lambda)]
$$

which shows that the potential $\phi(r)$ must be a bounded function of $r$, defined up to an arbitrary constant. Note also that Eq.(2.12) has the form of the potential energy of a pointlike scalar charge of value $2 \Lambda$ placed at infinity in the soliton field.

There is another way round to obtain the expression (2.12). Indeed, by performing the usual reescaling of Derrick's theorem [10], i.e. by defining a uniparametric family of transformations

$$
\phi_{\lambda}(x) \equiv \phi(\lambda x)
$$

3 In some cases Eq.2.4 can lead to discontinuities or several branches for the function $\phi^{\prime}(r)$. We shall regard such cases as "unphysical" and rule them out from this analysis, considering only models for which the fields of the SSS solutions are (for $r>0$ ) continuous, single-branched functions defined everywhere. We shall establish at the end of this section that the corresponding admissibility condition for the lagrangian densities is the strict monotonicity. 
it can be easily checked that the condition of extremum of the energy against these reescalings $\left(\left.\frac{d \epsilon(\phi(\lambda x))}{d \lambda}\right|_{\lambda=1}=0\right)$ leads automatically to the relation (2.12) when the condition of finiteness of the energy is assumed. Although Derrick's theorem is only a necessary condition for stability, this signals the presence of a connection between stability and finite-energy condition of the SSS solutions considered here. This connection will be precisely established in section 6.

Going beyond the results of reference [34], we shall determine general conditions to be imposed on the functions $f(X)$ in order to obtain physically consistent field theories, whose associated SSS solutions be stable and their energy (2.10) be finite (non-topological solitons). We first summarize some criteria of physical consistency adopted for the purposes of the present study (defining what we shall call "admissible" field theories) and obtain the associated restrictions on the lagrangian densities. Next we shall obtain the conditions for such admissible models to support SSS soliton solutions.

\subsection{Conditions on the energy functional}

If any of these models are to be used for descriptions of quantum physical systems, the possibility of their quantization becomes important. This implies supplementary conditions to be satisfied by the lagrangian densities, as necessary for any quantum extension. Thus, aside from the above mentioned continuity and derivability conditions, we must require the function $f(X)$ to be defined everywhere $(\Omega \equiv \Re)$, in order to allow the proper definition of the associated path integral. Here we shall call such models class-1 field theories 4.

Alternatively, as discussed in the introduction, we can consider these scalar models (and their generalized versions proposed below) as effective classical lagrangians of more "fundamental" theories, including integrated high-energy and quantum effects through new nonlinear couplings. In these cases we can relax the everywhere definiteness conditions of the admissible lagrangians and require their regularity only within a restricted domain of definition $(\Omega \subset \Re)$, which is assumed to be open, connected and including the vacuum $(0 \in \Omega)$. We shall call these models class-2 field theories. Using this criterion, models such as the BornInfeld one become admissible field theories belonging to this class. Although these models are essentially classical, quantum corrections to their particle-like solutions can be obtained by quantizing the field of small fluctuations around these ground states. Such fluctuations obey Euler-Lagrange linear field equations of admissible lagrangian densities defined everywhere (see Ref. [39] and section 6, Eqs. (6.11) and (6.12) below).

Obviously, a second condition to be imposed for admissibility in all cases concerns the positive definite character of the energy, which is required to hold in the entire domain of definition $(\Omega)$ of the lagrangian density. The expression for the energy density in terms of $f(X)$ is

\footnotetext{
4 These conditions exclude models such as the scalar version of Born-Infeld Electrodynamics [3]. In such cases it is always possible to generalize the model, by continuing the lagrangian density function to the undefined regions through some prescription which must preserve the classical dynamical content of the initial model. Then, the quantum behaviour of the extended model would depend on this prescription. But this procedure will necessarily enlarge the space of solutions of the classical theory. As we shall show below, these extensions introduce new branches for the SSS solutions which become spurious at the classical level. We shall exclude such extended models from the present analysis since they are non-admissible according to our physical criteria.
} 


$$
\rho=T^{00}=2 \dot{f}(X)\left(\frac{\partial \phi}{\partial t}\right)^{2}-f(X)=2 X \dot{f}(X)-f(X)+2 \dot{f}(X)(\vec{\nabla} \phi)^{2} .
$$

For the D'Alembert lagrangian we have $f(X)=X / 2$, and the energy density reduces to

$$
\rho=\frac{1}{2}\left[\left(\frac{\partial \phi}{\partial t}\right)^{2}+(\vec{\nabla} \phi)^{2}\right]
$$

which is positive for any non-constant function $\phi(t, \vec{r})$ and vanishes in vacuum. In order to obtain a similar behaviour in the general case (2.14) (requiring also the energy density to vanish in vacuum) we are lead to the necessary conditions

$$
\begin{gathered}
f(0)=0 \\
\stackrel{\bullet}{f}(X) \geq 0 \quad \forall X .
\end{gathered}
$$

For minimal sufficient conditions on $f(X)$ let us analyze separately the cases $X<0$ and $X>0$. On the one hand for $X<0$ the term $\left(\partial_{t} \phi\right)^{2}$ may vanish and the positivity of the energy density (2.14) requires

$$
f(X)<0 \quad(\forall X<0) .
$$

On the other hand, for $X>0$ the term $(\vec{\nabla} \phi)^{2}$ may vanish and the positivity of the energy requires

$$
\rho(X) \geq 2 X \dot{f}(X)-f(X)=X f(X) \frac{d}{d X}\left[\ln \left(\frac{f^{2}(X)}{X}\right)\right] \geq 0 .
$$

This equation, together with the conditions $f(0)=0$ and $\stackrel{\bullet}{f}(X) \geq 0(\forall X)$, lead to

$$
\frac{d}{d X}\left[\ln \left(\frac{f(X)}{\sqrt{X}}\right)\right] \geq 0
$$

or, equivalently, the function $\frac{f(X)}{\sqrt{X}}$ (and hence $f(X)$ itself) must be a positive monotonically increasing function of $X$ for $X>0$. Equation (2.19), together with the boundary condition $f(0)=0$, fix the behaviour of $f(X)$ around $X=0$ as

$$
f\left(X \rightarrow 0^{+}\right) \sim X^{1+\alpha},
$$

with $\alpha>-1 / 2$, and the energy density behaves there as 5

$$
\rho\left(X \rightarrow 0^{+}\right) \geq(1+2 \alpha) X^{1+\alpha} .
$$

\footnotetext{
5 For values of $\alpha$ in Eqs. (2.20) and (2.21) which lie in the interval $-1<\alpha<-1 / 2$ the condition $f(0)=0$ is fulfilled, but $\dot{f}(X)$ diverges at $X=0$ in such a way that the energy density becomes necessarily negative in the neighbourhood of $X=0$. For $\alpha \leq-1, f(X)$ diverges in vacuum. The limit case $\alpha=-1 / 2$ is singular.
} 


\subsection{Conditions for finite-energy SSS solutions}

The convergence of the integral of energy (2.10) for the SSS solutions is governed by the behaviour of the integrand near the limits $r \rightarrow \infty$ and $r \rightarrow 0$. This imposes supplementary conditions on the form of the function $f(X)$ around the values of $X(r)=-\phi^{\prime 2}(r)$ in these limits. Let us assume a power law expression for the field around these regions $6\left(\phi^{\prime}(r) \sim r^{q}\right.$ as $r \rightarrow \infty$ or as $r \sim 0$ ); from the first-integral (2.4) we obtain the relation (valid for $q \neq 0$; for $q=0$ at $r=0$ see the discussion of the case $\mathbf{A - 2}$ below)

$$
d f / d r=-\frac{2 \Lambda \phi^{\prime \prime}(r)}{r^{2}} \sim-2 \Lambda q r^{q-3}
$$

and in the limits of integration, $f(r)$ behaves as

$$
f(r) \sim \frac{2 \Lambda q}{2-q} r^{q-2}+D
$$

if $q \neq 2$, or as

$$
f(r) \sim-4 \Lambda \ln (r)+D
$$

if $q=2$. The integration constants $D$ in these expressions are easily related to the values of $X$ and $f(X)$ on the limits of the integral of energy Eq.(2.10). Around each of these limits the contributions to this integral take the form

$$
-4 \pi \int d r\left[\frac{2 \Lambda q}{q-2} r^{q}+D r^{2}\right],
$$

for $q \neq 2$ and

$$
-4 \pi \int d r\left[4 \Lambda r^{2} \ln (r)+D r^{2}\right]
$$

for $q=2$. Let us analyze separately the convergence of the energy integral around $r \sim 0$ (case A) and in the asymptotic limit $r \rightarrow \infty$ (case B). In case $\mathbf{A}$ the convergence of $(2.25)$ requires $q>-1$ and we can distinguish three sub-cases:

- $\underline{\mathbf{A}-1}$ ) If $-1<q<0$ the field $\phi^{\prime}(r)$ diverges at $r \rightarrow 0$ but the integral of energy converges there and the potential $\phi(r)$ is finite at the origin. Then, as $r$ approaches zero, $X \rightarrow-\infty$ and $f(X)$ and $\dot{f}(X)$ diverge as

$$
f(X) \sim-(-X)^{\frac{q-2}{2 q}}, \dot{f}(X) \sim(-X)^{-\frac{q+2}{2 q}},
$$

(see figure 1). Such solutions can be stable and finite-energy SSS fields (depending on their large- $r$ behaviour) and, in this sense, they might be considered as genuine non-topological solitons.

6 Although this assumption excludes some transcendent behaviours such as the asymptotic exponential damping, our conclusions will remain valid for models exhibiting these "short-ranged" SSS solutions. In fact such models are included in the case B-3 below. Note that damped oscillatory behaviour at infinity is excluded by the monotonicity of the SSS field solutions. 


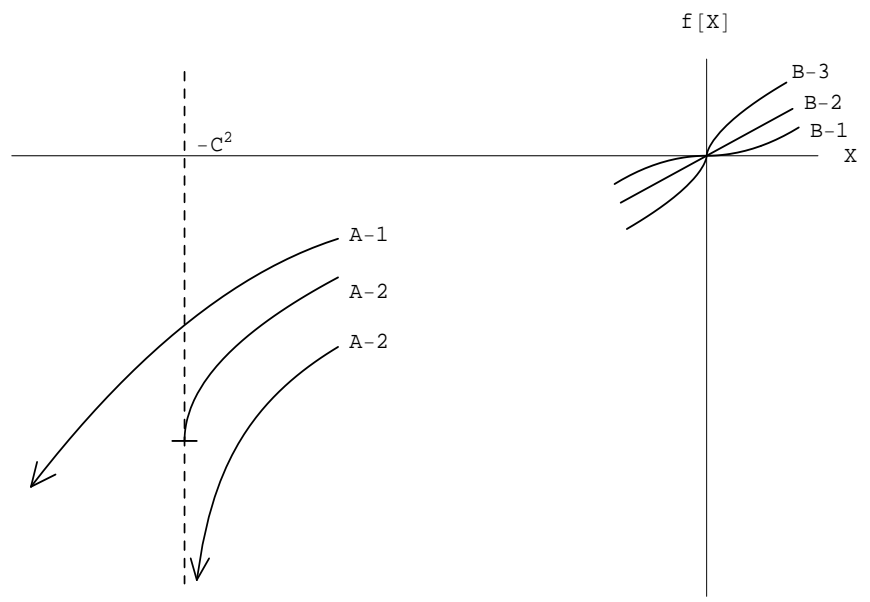

Fig. 1. Different possible behaviours of the admissible lagrangians supporting finite-energy SSS solutions. Note the existence of two A-2 cases, both ending at a finite value $X=-C^{2}$ (corresponding to the maximum value of the field strength) but with bounded or unbounded values of $f(X)$ there. A-1 branch is parabolic with divergent slope as $X \rightarrow-\infty$.

- $\underline{\mathbf{A}-2})$ If $q=0$ the field $\phi^{\prime}(r)$ goes to a constant value at the origin $\left(\phi^{\prime}(0)=C\right.$, which corresponds to $X+C^{2}=0$ ) and can be written around this point as

$$
\phi^{\prime}(r) \sim C-\theta r^{\sigma}
$$

where $C, \theta$ and $\sigma$ are positive constants 7 . Then $\dot{f}(X)$ diverges there as

$$
\dot{f}(X) \sim\left(X+C^{2}\right)^{-\frac{2}{\sigma}}
$$

Consequently, for $\sigma \neq 2, f(X)$ behaves around $X=-C^{2}$ as

$$
f(X) \sim \frac{\left(X+C^{2}\right)^{1-\frac{2}{\sigma}}}{\left(1-\frac{2}{\sigma}\right)}+\Delta,
$$

where $\Delta$ is a constant. For $\sigma=2$ this behaviour becomes

$$
f(X) \sim \ln \left(X+C^{2}\right) .
$$

We see that when $\sigma \leq 2$ these lagrangians diverge at $X=-C^{2}$ (figure 1). However they can be accepted as admissible class- 2 field theories if this point is located on the frontier of the (open) domain of definition where the lagrangian density must be regular (see the third example of the following section). Consequently the set $X \leq-C^{2}$ must be excluded from the domain of definition $(\Omega)$ in this case. This requires $\sigma$ to be an irrational number or four times the irreducible ratio between any natural and an odd natural. When $\sigma>2$ these values also exclude the same region, leading again to admissible class-2 field theories. However, in this case the lagrangians are finite in $X=-C^{2}$ (even if $\dot{f}(X)$ diverges there). Then, for rational values of $\sigma>2$ which are irreducible ratios of an odd natural and any natural 8, the lagrangians are defined and continuous for any $X$ around $X=-C^{2}$, exhibiting a

\footnotetext{
7 Note that the scalar version of the Born-Infeld model is an example which belongs to this case, corresponding to $\sigma=4$.

8 Other values of $\sigma$, for which the lagrangian is also defined for $X<-C^{2}$, lead to negative energy densities there and consequently must be excluded.
} 
vertical-slope inflexion in this point. If appropriately extended to all $X$, they lead to class1 field theories which satisfy the everywhere positive definiteness condition of the energy. Nevertheless they violate the requirement of continuity of $\dot{f}(X)$ for $X<0(\in \Omega)$ and this leads to associated point-like solutions exhibiting several branches. Indeed, as we shall see at once, this requirement for admissibility is introduced because it endorses the single-branched character of the SSS solutions. Consequently, in this case A-2 all admissible lagrangians must remain undefined for $X \leq-C^{2}$ and thus belong to class-2 field theories. This implies (as happens in the Born-Infeld model [3]) the existence of a maximum value of the field strength $\left(\phi^{\prime}(r)<C\right)$.

As a function of $r$, the energy density behaves around the center as

$$
r^{2} f(r) \sim \frac{(2 C \theta)^{1-2 / \sigma}}{1-2 / \sigma} r^{\sigma}-\Delta r^{2},
$$

for $\sigma \neq 2$ and as

$$
r^{2} f(r) \sim 2 r^{2} \ln (r)+r^{2} \ln (2 C \theta),
$$

for $\sigma=2$. As expected, the energy integral converges there in both cases.

- $\underline{\mathbf{A}-\mathbf{3}})$ The case $q>0$ must be discarded. Indeed, in this case $\dot{f}(X)$ behaves as

$$
\dot{f}(X) \sim X^{-\frac{q+2}{2 q}},
$$

around $X=0$. Consequently, $f(X)$ is singular in vacuum for $0<q \leq 2$. For $q>2$ the energy density for $X \rightarrow 0^{+}$behaves as

$$
\rho(X) \sim-\frac{2}{q} X^{\frac{q-2}{2 q}}
$$

and becomes negative around the vacuum (see also Eqs.(2.20), (2.21) and the footnote there).

In case $\mathbf{B}$ the convergence of (2.25) in the $r \rightarrow \infty$ limit requires $q<-1$ (in this case $\phi^{\prime}(r \rightarrow \infty)=0$ and the integration constant $D$ in (2.25) vanishes). Then the behaviour of $f(X)$ around $X=0$ must be 9

$$
f(X) \sim X^{\frac{p+2}{2 p}} .
$$

Now the existence of the lagrangian on both sides around $X=0$ becomes crucial for the consistency of the theory and this imposes supplementary restrictions on the possible values of the parameter $p$. Indeed, the exponent in Eq.(2.36) must be the ratio of two odd naturals 10 and this restricts the possible values of $p$ to a sub-class of the rational numbers. Let us analyze separately three possibilities:

- $\underline{\mathrm{B}-1})$ Consider first the case $1<p<2$. We define $P$ and $Q$ as two positive odd natural numbers in such a way that $P<Q$ and the ratio $\Sigma=P / Q$ be irreducible. Then the admissible values of the exponent in (2.36) are given by

$$
\frac{p+2}{2 p}=\frac{3}{2+\Sigma}
$$

9 For the sake of clarity we use here the parameter $p=-q$ in the exponent, in terms of which $\phi(r \rightarrow \infty) \sim \frac{1}{r^{p}}$ with $p>1$. ${ }^{10}$ If this exponent is the irreducible ratio between an even and an odd natural numbers the lagrangian is well defined on both sides of $X=0$, but the energy density becomes negative for $X<0$. 
and the corresponding admissible values of $p$ can be written as

$$
p=\frac{4+2 \Sigma}{4-\Sigma} .
$$

Now $\dot{f}(0)=0$ and the slope of the lagrangian vanishes in vacuum (see figure 1 ).

- $\underline{\mathbf{B}-2})$ For $p=2$ the lagrangian behaves around $X=0$ as the D'Alembert lagrangian

$$
f\left(X \rightarrow 0^{ \pm}\right) \sim X
$$

and the soliton field becomes asymptotically coulombian (see figure 1).

- $\underline{\mathbf{B}-3})$ For $p>2$ the behaviour of the lagrangian is also given by Eq.(2.36) but now the admissible values of the exponent are constrained by

$$
\frac{p+2}{2 p}=\frac{1}{1+\Sigma}>\frac{1}{2}
$$

where $\Sigma=P / Q$ must be the irreducible ratio between an even natural $P$ and an odd natural $Q$ such that $Q>P$. The corresponding admissible values of $p$ are

$$
p=2 \frac{1+\Sigma}{1-\Sigma} \text {. }
$$

As easily seen, the slope of the lagrangian diverges at $X=0$ in this case (see figure 1 ), but the energy density remains positive definite there.

We conclude that the set of admissible models exhibiting finite-energy SSS solutions can be classified into six families which are the combinations of the cases A-1 or A-2, governing the central field behaviour and the cases B-1, B-2 or B-3, determining the asymptotic field behaviour. Moreover, any given scalar, monotonically decreasing, SSS function $\phi^{\prime}(r)$, which satisfies boundary conditions of A-type at the center and of B-type asymptotically, is a finiteenergy SSS solution of a particular lagrangian model belonging to one of these families. The explicit form of the corresponding lagrangian density can be found by integrating Eq.(2.4) with respect to the variable $X=-\phi^{\prime 2}(r)$ with the corresponding boundary conditions (see the fourth example of the next section).

\subsection{Conditions for stability}

We summarize here the main steps in the analysis of linear stability of the scalar SSS soliton solutions (the detailed calculations are given in section 6). The linear stability of these solutions requires their energy (2.10) to be a local minimum against small charge-preserving perturbations. We consider finite-energy SSS solutions $\phi(r)$ and small static perturbations $\delta \phi(\vec{r})$, finite and regular everywhere and vanishing (as well as their radial derivatives) as $r \rightarrow \infty$, in such a way that the scalar charge of the perturbed fields remains unchanged at the first order in the perturbations. For the static solutions of the field equations (2.2) the first variation of the energy (2.14) vanishes, while the second variation is positive if and only if the condition 11

$$
\dot{f}(X)+2 X \ddot{f}(X) \geq 0,
$$

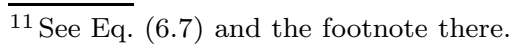


is satisfied in all the range of values of $X$ covered by the solution $\left(X=-\phi^{\prime 2}(r), 0 \leq r<\right.$ $\infty)$. As we shall see this requirement is always fulfilled by the finite-energy SSS solutions of the admissible models defined in this section, proving their linear stability. A detailed spectral analysis of the small oscillations around these SSS finite-energy solutions has also been performed. It leads, for admissible models, to discrete spectra of eigenvalues and normalizable orthogonal eigenfunctions in Hilbert spaces, whose scalar products are built as three-dimensional integrals of their products with the functions $f(X(r))$ as kernels. In their temporal evolutions the perturbations to the soliton solutions remain bounded in this norm, confirming the stability (see subsections 6-1 and 6-2).

\subsection{Conditions for uniqueness of the elementary solutions}

We return now to Eq.(2.4), which defines the SSS field solutions $\phi^{\prime}(r)$, and analyze the conditions under which they are single-branched and defined everywhere functions. As already mentioned, owing to the positivity of $\dot{f}(X)$ both $\Lambda$ and $\phi^{\prime}(r)$ must be simultaneously either positive or negative and then we can analyze only the case $\phi^{\prime}(r)>0$ without loss of generality. Let us write (2.4) under the form

$$
z(y)=y \dot{f}\left(-y^{2}\right)=\frac{\Lambda}{r^{2}} \geq 0
$$

where we have introduced the variable $y=\phi^{\prime}(r)>0$. The field strength function is given by the values of $y$ obtained by cutting the curve $z(y)$ with horizontal lines corresponding to the different values of $r$. Then, for the field $\phi^{\prime}(r)$ to be defined in all space, $z$ must range from 0 to $\infty$ and the uniqueness of the solution requires a single cut point on every $z=$ constant line. This restricts $z(y)$ to be a continuous monotonic function. As a consequence, the requirements of continuity (for $X<0$ ) and strict positivity (for any $X \neq 0$ ) imposed on $\dot{f}(X)$ at the beginning of this section, when establishing the admissibility conditions, are mandatory for the proper definition of the SSS solutions. Indeed, a glance at the form of the function $z(y)$ reveals that the existence of a jump in the function $\dot{f}(X)$ at a finite value $X<0$ would lead to SSS solutions which are double-branched or undefined in some range of values of $r$. Moreover, if $\dot{f}(X)$ vanishes for a value $X_{0}<0$ (horizontal-slope inflexion point for $f(X))$, then $z(y)$ vanishes at $y=\left(-X_{0}\right)^{1 / 2}$ reaching a minimum there and the SSS solutions become necessarily multiple-valued (the vanishing of $f(X)$ for a positive value $X_{0}>0$ is discarded by the energy-positivity condition (2.19)).

For soliton solutions the finiteness of the energy requires the origin to be a point of the curve $z(y)$ (see figure 2) and the large- $z$ behaviour is determined by the behaviour of the field at the center of the soliton. Then, in the cases where the field strength diverges as $r \rightarrow 0$ (case A-1 above) the uniqueness of the solution requires the curve $z(y)$ to start at the origin and grow monotonically without limit as $y \rightarrow \infty$. When the field is finite at $r=0$ (case A-2) the curve $z(y)$ must increase monotonically from the origin and diverge at $y_{0}=\phi^{\prime}(0)$, showing a vertical asymptote there. In this case $\dot{f}\left(-y^{2}\right)$ diverges at $y=y_{0}$ and the uniqueness condition requires the lagrangian function to remain undefined for $y>y_{0}$, for 


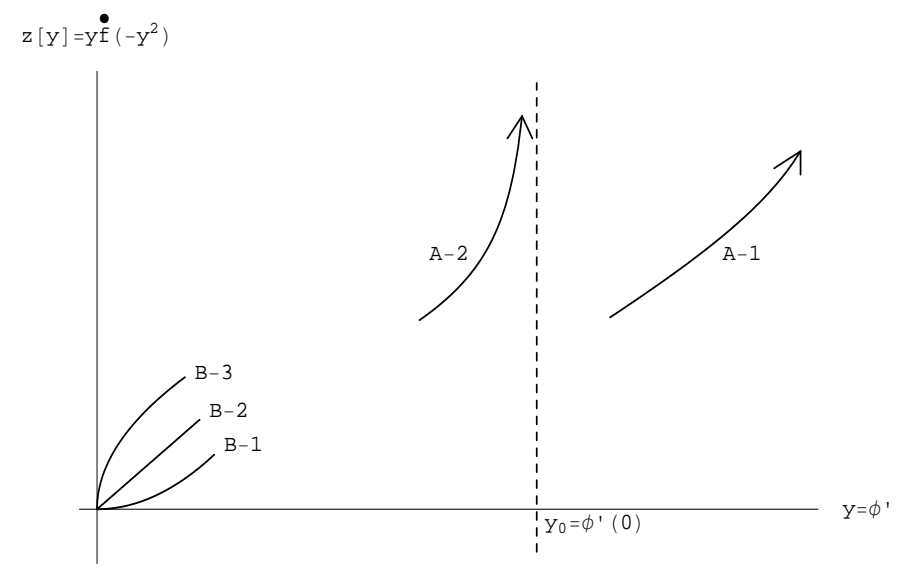

Fig. 2. Characterization in the $y-z(y)=y \dot{f}\left(-y^{2}\right)$ plane of the admissible models supporting finite-energy SSS solutions. Observe that all curves start at $y=0, z=0\left(y=\phi^{\prime}(r \rightarrow \infty)=0\right.$, cases B-1, B-2 and B-3) and grow monotonically without limit. This guarantees the existence of a single cut point with the $z=$ constant lines and thus a single-valued solution (compare to figure 1).

the function $z(y)$ to exhibit an unique growing branch. Thus in all A-2 cases the set $X<-y_{0}^{2}$ must be excluded from the domain of definition $(\Omega)$ and then, the associated models must be necessarily class-2 field theories 12 . The monotonicity condition for the unique branch of $z(y)$ in the admissible models with finite-energy solutions takes the form

$$
\frac{d z}{d y}=\dot{f}\left(-y^{2}\right)-2 y^{2} \ddot{f}\left(-y^{2}\right) \geq 0,
$$

for any $y \geq 0$. This requirement coincides with the condition (2.42) for stability which, as already mentioned, is fulfilled by all these admissible models. To summarize, we conclude that all finite-energy SSS solutions of admissible scalar field theories considered in this section are single-branched, stable and defined everywhere.

To close this section let us give an expression for the energy of the SSS solutions in terms of the function $z(y)$, which will be useful for the explicit calculation of the soliton energy, as we shall see in the examples of the next section. This expression can be obtained by taking into account Eqs.(2.4), (2.11) and (2.43) and reads

$$
\epsilon=\frac{4 \pi}{3} \Lambda^{3 / 2}\left\{\left.\frac{f\left(-y^{2}\right)}{z(y)^{3 / 2}}\right|_{y(r=0)} ^{y(r \rightarrow \infty)}-2 \int_{y(r=0)}^{y(r \rightarrow \infty)} \frac{d y}{\sqrt{z(y)}}\right\} .
$$

As can be seen from the preceding analysis, the first term in this formula vanishes for admissible models with soliton solutions 13 . Once the expression of the lagrangian density is known, the second term gives the soliton energy directly through a quadrature.

\footnotetext{
${ }^{12}$ It can be shown from the analysis of the possible continuations of $z(y)$ for $y>y_{0}$ that the new branches of SSS field solutions are pathological (non-defined everywhere, unstable, or both).

${ }^{13}$ Conversely, the conditions to be imposed on the lagrangian densities of admissible models to support finite-energy SSS solutions could have been directly obtained from the requirement of cancellation of the first term in (2.45).
} 


\section{Some examples}

As illustrative examples of the considerations of the previous section we shall introduce and discuss four families of admissible models representative of the different classes analyzed there.

\subsection{Potential corrections to the D'Alembert Lagrangian}

The first example is given by the two-parameter family of field theories defined by lagrangian densities of the form

$$
f(X)=\frac{X}{2}+\lambda X^{a}
$$

where $\lambda$ is a positive constant which gives the intensity of the self-coupling. When $\lambda=0$, $f(X)$ reduces to the usual D'Alembert lagrangian density (see figure 3 ). The values of the exponent $a$ are restricted to be irreducible ratios of two odd natural numbers (we consider 1 as odd) $a=P / Q$ such that

$$
P>\frac{3}{2} Q\left(\Rightarrow a>\frac{3}{2}\right)
$$
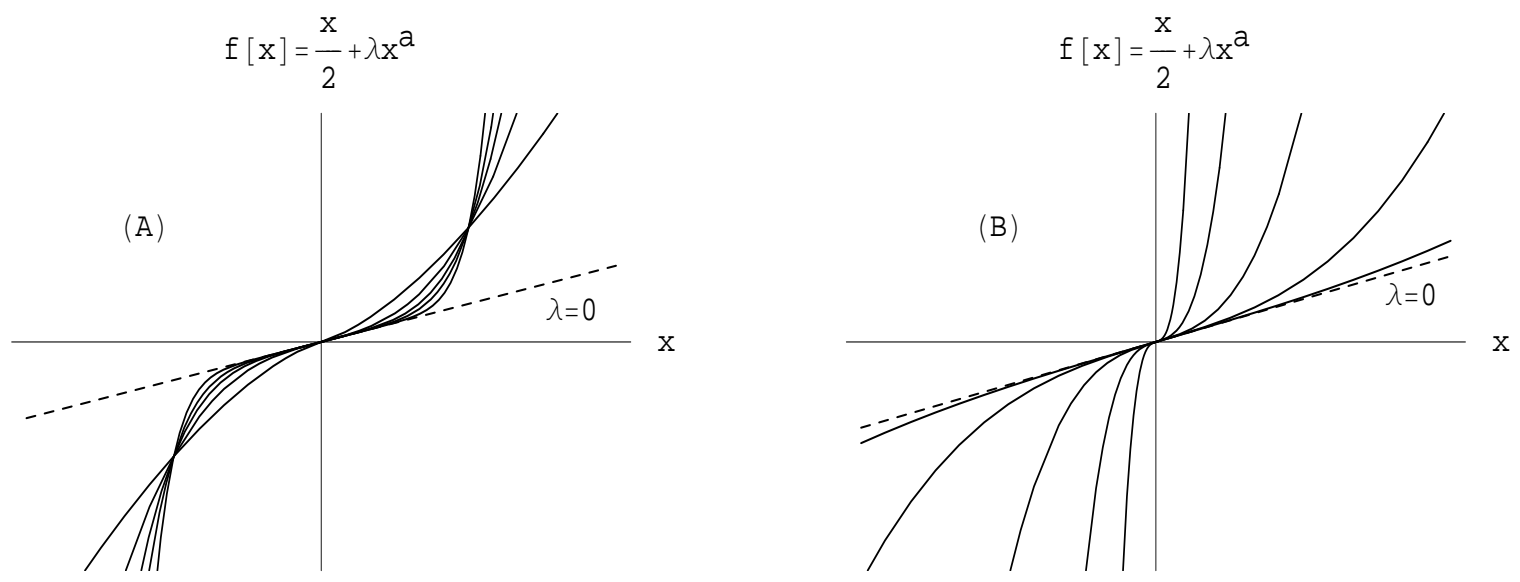

Fig. 3. Functional form of the lagrangian densities for the family of models 3.1) (A) for a fixed value of the parameter $\lambda(=1)$ and several values of $a(=5 / 3,3,13 / 3,17 / 3,25 / 3)$ (B) for a fixed value of the exponent $a(=3)$ and several values of $\lambda(=0.01,0.1,1,10,100)$. The dashed line corresponds in both cases to the D'Alembert lagrangian $(\lambda=0)$.

As easily verified these restrictions allow $f(X)$ to be defined everywhere and the associated energy density to be positive definite and vanishing in vacuum (admissible class-1 field theories). The condition $a>3 / 2$ is imposed to ensure the convergence of the integral of energy at $r=0$, where the SSS field strengths diverge.

This family of models can be extended to include rational values of the exponent $a=$ $P / Q>3 / 2$ with $P$ being an even natural number and $Q$ an odd one. Such models are 
admissible for $X<0$ if we replace $\lambda>0$ by $-\lambda$ in Eq.(3.1) 14 . The following considerations are valid for the extended family. The form of the SSS solutions is obtained from the equation (2.44), which now reads

$$
z(y) \equiv \frac{y}{2}+(-1)^{P-1} \lambda a y^{(2 a-1)}=\frac{\Lambda}{r^{2}},
$$

with $y(r, \Lambda) \equiv \phi^{\prime}(r, \Lambda)$. The function $z(y)$ shows an unique growing branch for every value of the scalar charge $\Lambda>0$ and, consequently, there is an unique SSS solution of Eq.(3.3), which vanishes as $\phi^{\prime} \sim r^{-2}$ when $r \rightarrow \infty$ (case B-2 above, asymptotically coulombian) and diverges as $\phi^{\prime} \sim r^{-2 /(2 a-1)}$ when $r \rightarrow 0$. Thus the argument $(X)$ of the lagrangian ranges from zero to $-\infty$ in this interval and, as expected, the stability condition (2.42), which now reads

$$
\stackrel{\bullet}{f}(X)+2 X \stackrel{\bullet}{f}(X)=\frac{1}{2}+(-1)^{P-1} \lambda a(2 a-1) X^{a-1}>0, \quad \forall X<0,
$$

is fulfilled there.

The energy of the soliton, as a function of the model parameters, can be explicitly obtained from the integral term of Eq.(2.45). The final result is

$$
\epsilon=\frac{4 \sqrt{2} \pi}{3} \frac{\Lambda^{3 / 2}}{(a-1)(2 a \lambda)^{\frac{1}{4(a-1)}}} B\left(\frac{1}{4(a-1)}, \frac{2 a-3}{4(a-1)}\right),
$$

where $B(x, y)$ is the Euler integral of first kind

$$
B(x, y)=\int_{0}^{1} d t t^{x-1}(1-t)^{y-1}, \quad \operatorname{Re}(x)>0, \quad \operatorname{Re}(y)>0 .
$$

In figure 4 we have plotted this energy as a function of the exponent $a$, with the coupling constant $\lambda$ as a parameter. We see that the energy diverges, for any value of $\lambda$, as the exponent $a$ approaches the value $3 / 2$. This energy is strongly reduced in the region of values of $a \gtrsim 3 / 2$ as the coupling constant $\lambda$ increases, reaching minima which vanish as $\lambda \rightarrow \infty$ in this region. When the exponent $a$ increases the energies of the solitons become less dependent on $\lambda$ and approach asymptotically the value $\epsilon / \Lambda^{3 / 2}=16 \pi \sqrt{2} / 3$ as $a \rightarrow \infty$. An interesting feature of these models is the existence of soliton solutions for any $a>3 / 2$, no matter how small the coupling parameter $\lambda$ may be. This implies that any small correction of this kind to the "bare" D'Alembert lagrangian leads to the possibility of excitation of soliton modes. For a fixed value of the exponent, the masses of such modes increase as the intensity of the coupling is reduced. This behaviour is similar to the one encountered in one-space dimensional models supporting topological soliton solutions [39], [40].

The preceding analysis can be generalized to the case of theories whose lagrangian densities for $X<0$ take the form 15

\footnotetext{
${ }^{14}$ But, for complete admissibility, this function should be matched for $X>0$ with another function satisfying the condition (2.19). For example, the lagrangian obtained by replacing $\lambda$ by $\lambda \cdot \operatorname{sign}(X)$ in (3.1). Since the structure and energy of the SSS solutions are determined by the form of the lagrangian density for $X<0$, the SSS solutions of these models are also solitons.

${ }^{15}$ Obviously, for $X>0$ they must be extended in agreement with the requirements of section 2 .
} 


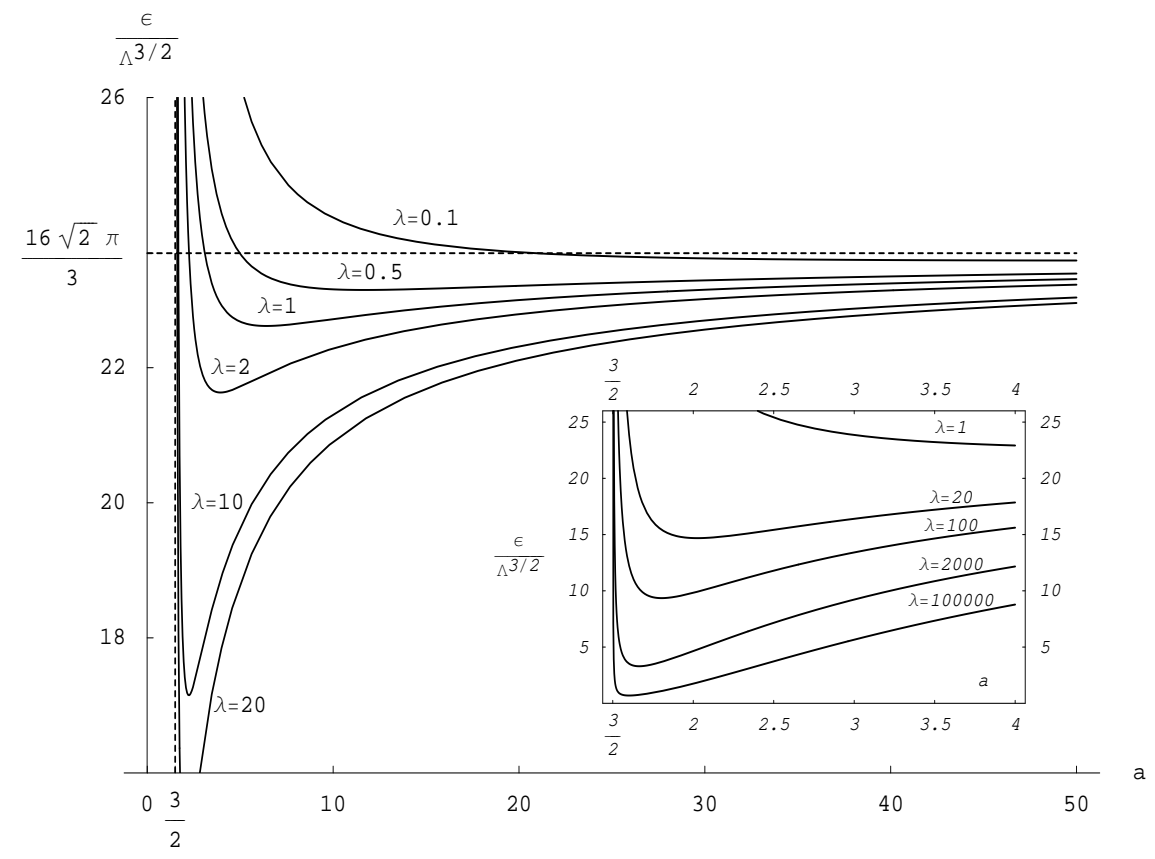

Fig. 4. Energy of the solitons of the family (3.1) as a function of the exponent $a$ with the coupling constant $\lambda$ as parameter. The dashed line correspond to the asymptotic limit of the energy for $a \rightarrow \infty$. The small plot shows the behaviour of the energy for strong self-couplings. The energy is never zero for any $\lambda<\infty$.

$$
f_{N}(X)=\frac{X}{2}+\sum_{n=2}^{N}(-1)^{P_{n}-1} \lambda_{n} X^{a_{n}},
$$

where, for admissibility, the $\lambda_{n}$ are constrained to form a finite sequence of positive constants and the exponents $a_{n}=P_{n} / Q_{n}$ to form an increasing sequence of rational numbers, built as irreducible ratios of odd natural numbers or of even and odd naturals, such that $a_{n}>1$ for $n<N$ and $a_{N}>3 / 2$. In these models the energy density is positive definite for $X<0$ whereas the SSS field solutions diverge at the origin as $\phi^{\prime}(r \rightarrow 0) \sim r^{-2 /\left(2 a_{N}-1\right)}$ and vanish asymptotically as $\phi^{\prime}(r \rightarrow \infty) \sim r^{-2}$. The associated energies are finite and the corresponding solitons are stable.

A particular case of Eq.(3.7) is obtained when the exponents are a finite sequence of consecutive naturals $a_{n}=n(n>1)$. Moreover, let us assume that we take the limit $N \rightarrow \infty$ and that the infinite sequence of $\lambda_{n}>0$ converges to zero, in such a way that the series

$$
f(X)=\frac{X}{2}+\sum_{n=2}^{\infty}(-1)^{n-1} \lambda_{n} X^{n},
$$

be convergent in an interval including $\forall X<0$ (case A-1), or in an interval including the range $0>X>-C^{2}$ but excluding the values $X<-C^{2}$ (case A-2). We can then extend the family (3.7) to a large class of analytic functions. Such functions must satisfy the conditions established in section 2.2. for the integrals of energy associated to the SSS solutions of such models to be finite. If we assume the positivity of the coefficients $\lambda_{n}$ in Eq.(3.8) we can explicitly check for these models the fulfillment of the condition of stability (2.42), which now takes the form 


$$
\dot{f}(X)+2 X \ddot{f}(X)=\frac{1}{2}+\sum_{n=2}^{\infty} n(2 n-1) \lambda_{n}(-X)^{n-1}>0
$$

and holds in the entire domain of definition of the soliton. Thus the requirement of convergence of the series (3.8) for any $X$, with the assumed restrictions $\lambda_{n}>0(\forall n)$ leads to class-1 field theories supporting SSS solitons. As an example of this let us mention the analytic function $f(X)=\frac{1}{2} \operatorname{sh}(X)$, which diverges when $X \rightarrow-\infty$ faster than $X^{\gamma}$ with $\gamma>3 / 2$ (as required by Eq.(2.27) in the A-1 case) and behaves like $X / 2$ around $X=0$ (case B-2).

If we relax the requirement of positivity of the coefficients $\lambda_{n}$, whenever the series (3.8) converges in some restricted interval $X>-C^{2}$ and the sum remains a monotonically increasing function of $X$ there, we are lead to admissible class-2 field theories exhibiting SSS soliton solutions. An example of this case is the lagrangian density $f(X)=\frac{1}{2} t g(X)$ restricted to the interval $-\frac{\pi}{2}<X<\frac{\pi}{2}$. This model supports finite-energy stable SSS solutions belonging to cases A-2 and B-2.

Let us consider the case $\lambda_{n} \geq 0, \forall n$. The partial sums in (3.8) give rise to an infinite sequence of admissible lagrangian models of the form (3.7) with natural exponents, all of them supporting SSS soliton solutions belonging to cases A-1 and B-2. The explicit forms $\phi_{N}(r, \Lambda)$ of these solutions can be obtained by solving the equation (2.43) for each lagrangian in the sequence. These equations take the form

$$
\phi_{N}^{\prime}\left(\frac{1}{2}+\sum_{n=2}^{N} n \lambda_{n}\left(\phi_{N}^{\prime}\right)^{2(n-1)}\right)=\frac{\Lambda}{r^{2}} .
$$

If the series (3.8) converges in $X<0$, defining an analytic lagrangian density function there, the sequence of SSS soliton solutions of the partial-sum models in the expansion, corresponding to the same value of the scalar charge $\Lambda$ for all $N$, must converge to the SSS soliton solution (with the same charge) associated to this lagrangian density $\left(\phi_{N \rightarrow \infty}(r, \Lambda) \rightarrow\right.$ $\phi(r, \Lambda))$. This can be directly established from Eq.(3.10), which defines the forms of the SSS solutions. Then the limit solution can be written as a functional series expansion in terms of the members of the sequence as

$$
\phi(r, \Lambda)=\phi_{1}(r, \Lambda)+\sum_{N=2}^{\infty} \delta_{N}(r, \Lambda)
$$

where $\delta_{N}(r, \Lambda)=\phi_{N}(r, \Lambda)-\phi_{N-1}(r, \Lambda)$. Using Eqs.(2.11) and (2.12) it is easy to show that the sequence of energies of the equal-charge solitons associated to the partial-sum lagrangian densities converges towards the energy of the equal-charge soliton associated to the full series lagrangian (3.8), which can be written as the series expansion:

$$
\epsilon(\Lambda)=\epsilon_{1}(\Lambda)+\sum_{N=2}^{\infty} \Delta_{N} \epsilon(\Lambda)
$$

where $\Delta_{N} \epsilon(\Lambda)=\epsilon_{N}(\Lambda)-\epsilon_{N-1}(\Lambda)$ is the difference between the energies of two consecutive solitons in the sequence. The first two terms of this expansion are energy-divergent. The first one corresponds to the divergent self-energy of the Coulomb field whereas the first correction $\Delta_{2} \epsilon(\Lambda)$ cancels this divergence and "renormalizes" the self-energy to a finite value. The subsequent terms are all finite and the series converges towards the energy of 
the limit soliton. The energy associated with the soliton of order $N$ in the sequence can be obtained making use of the expression (2.45) and reads

$$
\epsilon_{N}(\Lambda)=\frac{8 \pi}{3} \sqrt{2} \Lambda^{3 / 2} \int_{0}^{\infty} \frac{d y}{y^{1 / 2} \sqrt{1+2 \sum_{n=2}^{N} n \lambda_{n} y^{2(n-1)}}},
$$

which can be numerically calculated once the coefficients are fixed.

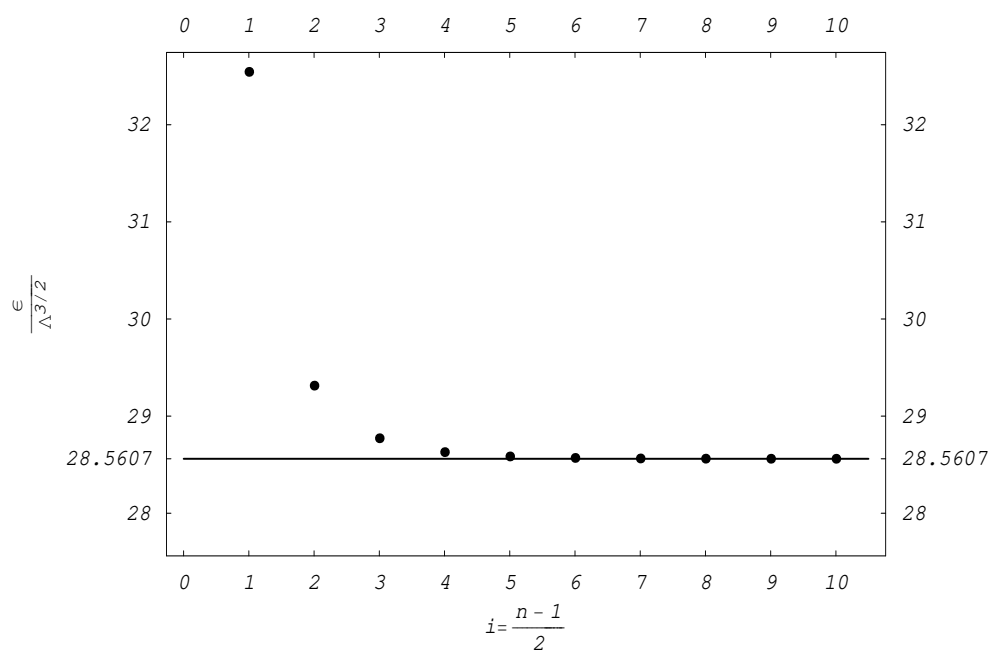

Fig. 5. Energy of the sequence of SSS soliton solutions (with the same scalar charge, $\Lambda$ ) of the partial-sum lagrangian models obtained from the McLaurin expansion of the lagrangian density $f(X)=\frac{1}{2} \operatorname{sh}(X)$, as functions of the integer parameter $i=\frac{N-1}{2}, N$ being the odd exponents of the surviving terms in the expansion. As $N$ increases these energies approach asymptotically the value $\epsilon(\Lambda) \simeq 28.5607 \Lambda^{3 / 2}$, corresponding to the soliton energy of the exact theory.

To illustrate this procedure let us consider the above mentioned analytic lagrangian $f(X)=\frac{1}{2} \operatorname{sh}(X)$. The energy associated to the soliton solutions of this model, obtained from Eq.(2.45), is $\epsilon(\Lambda) \simeq 28.5607 \Lambda^{3 / 2}$. The partial sums of the McLaurin expansion of this lagrangian function are admissible models supporting a sequence of SSS soliton solutions. Their energies, obtained from Eq.(3.13), are plotted in figure 5 as functions of $i=\frac{N-1}{2}$, for the same value of the scalar charge $\Lambda$ of each solution $(N=2 i+1$ being the exponents of the surviving terms in the expansion which, in this case, are the sequence of odd naturals). Obviously the energy of the first-order term $(N=1, i=0)$, which corresponds to the Coulomb field, diverges but, as expected, the first correction already "renormalizes" this coulombian divergent energy and the subsequent orders reduce the (now finite) energy, which approaches asymptotically the energy of the soliton of the exact model as $i$ increases. The convergence in this example is related to the analytic character of the sum (3.8) but the "renormalization" of the divergent self-energy is due to the first correction to the pure D'Alembert lagrangian and would arise even if the series were not convergent.

These results can be useful in the analysis of particle-like solutions in effective models of gauge-invariant interactions. Indeed, effective lagrangians arise frequently in perturbative schemas which lead to polynomial expressions in certain field invariants. For example, in the case of QED the perturbative expansion of the photon effective action, which is obtained by integrating out the high-energy degrees of freedom of the electron sector, defines a sequence 
of lagrangians which take this polynomial form in the field invariants (Euler-Heisenberg lagrangians [32] and the higher-order corrections [33]). On the other hand, as we shall establish in the following sections, the solution of the electrostatic spherically symmetric problem for a generalized gauge-invariant lagrangian model can be reduced to that of an associated scalar field model, whose lagrangian density is univocally defined from the gauge-invariant one. If the sequence of gauge-invariant effective lagrangians are of polynomial forms in the field invariants, the associated scalar lagrangians are also polynomials in the kinetic term, taking the form of partial sums of a series as (3.8). In this way we have established that the sequence of effective lagrangians describing the low-energy photon-photon interaction in QED support electrostatic point-like finite-energy solutions [41.

Let us give a tentative physical interpretation of these results. The non-linear terms in these effective lagrangians describe, at a classical level, a self-interaction of the gauge field mediated by the Dirac vacuum. The point-like solution of the "bare" Maxwell lagrangian is the Coulomb field, which has a divergent self-energy. The first non-linear correction term of the effective lagrangian (Euler-Heinsenberg) incorporates polarization effects of the vacuum on the classical field of the point charge, calculated to lowest order in a perturbative expansion. These screening effects "renormalize" the charge field, which becomes finite-energy. The subsequent corrections in the expansion describe higher-order approximations to the behaviour of the screening, but the finite-energy character of the screened fields is preserved to all orders. Unfortunately, the validity of this effective approach is limited to energies much lower than the electron mass [31] and is not accurate to describe the strong fields arising near the center of the particle-like solutions. Consequently, this tentative interpretation can not be maintained only on these grounds. For a more rigorous investigation this question should be considered starting from a different effective approach incorporating the vacuum polarization effects in presence of the strong fields of point-like charges. The analysis of this approach is currently in progress (see also the comments on this point in the last section).

\subsection{B-I-like models}

The second example is a two-parameter family of field theories defined by lagrangian densities of the form

$$
f(X)=\frac{\left(1+\mu^{2} X\right)^{\alpha}-1}{2 \alpha \mu^{2}},
$$

where $\mu$ is a real constant. The admissibility conditions require the values of the parameter $\alpha$ to be restricted to the range $1 / 2 \leq \alpha<1$. Indeed, if $1 \leq \alpha \leq 3 / 2$ the energy of the SSS solutions diverges around $r=0$. On the other hand if $\alpha>3 / 2$ the solution $\phi^{\prime}(r)$ is multivalued and the different branches are either unstable or energy-divergent. We also discard the models with $0<\alpha<1 / 2$, since the energy density in this case is not positive definite for $X>0$. Moreover, if $\alpha$ is a rational number built as the irreducible ratio of an even natural

and an odd natural numbers, the function $f(X)$ is defined everywhere, but $\dot{f}(X)$ changes sign in $X=-1 / \mu^{2}$ and the energy becomes negative for large negative values of $X$. Finally, if $\alpha$ is the irreducible ratio of two odd naturals we are lead to models which exhibit multi- 
branched SSS solutions 16 . Consequently, we must exclude the models with these values of the parameter $\alpha$ and restrict the family to the lagrangian densities which result from irrational values of $\alpha$ or rational values which are irreducible ratios of an odd and an even natural (always within the range $1 / 2 \leq \alpha<1$ ). In these cases the lagrangian densities are defined only for $X>-1 / \mu^{2}$ and behave as $X / 2$ around $X=0$, corresponding to class-2 field theories and cases A-2 and B-1. There are now unique, stable and finite-energy SSS solutions for each model, which are defined everywhere and fall inside the cases A-2 and B-2 (with the maximum field strength $\phi^{\prime}(0)=1 / \mu$, and coulombian behaviour at infinity). In the limits $\mu \rightarrow 0$ or $\alpha \rightarrow 1$ Eq.(3.14) reduces to the D'Alembert lagrangian density. The scalar Born-Infeld model is a member of this family, corresponding to the frontier value $\alpha=1 / 2$.

In calculating the energy of these soliton solutions as a function of the model parameters we evaluate the integral in (2.45), as in the previous example. The final expression is

$$
\epsilon=\frac{4 \sqrt{2} \pi}{3} \frac{\Lambda^{3 / 2}}{|\mu|^{1 / 2}} B\left(\frac{1}{2}, \frac{3-\alpha}{2}\right) .
$$

We see that, as a function of $\mu$ the soliton energy behaves like $\epsilon \sim \frac{1}{\sqrt{\mu}}$ and diverges as $\mu \rightarrow 0$ (D'Alembert limit), whereas it vanishes in the strong-coupling limit $\mu \rightarrow \infty$. This energy is not very sensitive to the exponent $\alpha$ in the range of admissible values and the behaviour for the whole family is similar to that of the scalar Born-Infeld model.

\subsection{A three-parameter family}

The third example is the three-parameter family of models defined by the lagrangian densities

$$
f(X)=\frac{1}{2} \frac{X^{\alpha}}{\left(1+\mu^{2} X\right)^{\beta}},
$$

where $\alpha$ is chosen as the irreducible ratio of two positive odd naturals (in order for the lagrangian to be well defined on both sides of $X=0$ ). The exponent $\beta$ must be chosen as a positive irrational number or as the irreducible ratio of an odd and an even natural numbers. In this way the lagrangian is defined only for $X>-1 / \mu^{2}$, thus avoiding a nonpositive definite character of the energy as well as a singularity inside the domain of definition 17 . These restrictions lead to a family of class-2 field theories belonging to the A-2 case, being examples of the sub-cases whose lagrangians diverge at $X \rightarrow-1 / \mu^{2}$ in the boundary of the domain of definition. The behaviours of the lagrangians around $X=0(r \rightarrow \infty$ for the SSS solutions) belong respectively to the cases B-1 $(\alpha>1)$, B-2 $(\alpha=1)$ or B-3 $(\alpha<1)$, corresponding to asymptotic dampings of the soliton field strengths which are slower than coulombian, coulombian or faster than coulombian, respectively (see figure 6).

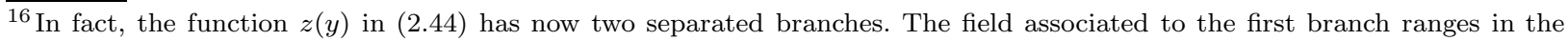
interval $0 \leq \phi^{\prime}(r)<1 / \mu$ and satisfies the condition (2.42) there, leading to a stable and finite-energy SSS solution, finite and defined everywhere (these branches fall inside the cases A-2 and B-2, with $\phi^{\prime}(0)=1 / \mu$ and coulombian asymptotic behaviour). The remaining branch of $z(y)$ ranges in the interval $1 / \mu<y<\infty$ and exhibits a minimum at $y=(\mu \sqrt{2 \alpha-1})^{-1}$. Consequently there are two additional solutions $\phi^{\prime}(r)$ defined only inside the interval $0 \leq r \leq \sqrt{2 \mu \Lambda}(2-2 \alpha)^{(1-\alpha) / 2}(2 \alpha-1)^{(2 \alpha-1) / 4}$.

${ }^{17}$ We emphasize that, as mentioned in section 2, we regard as acceptable singularities of the lagrangian density only those lying on the boundary of the (open and connected) domain of definition. In fact, one of the motivations in introducing this example
} 


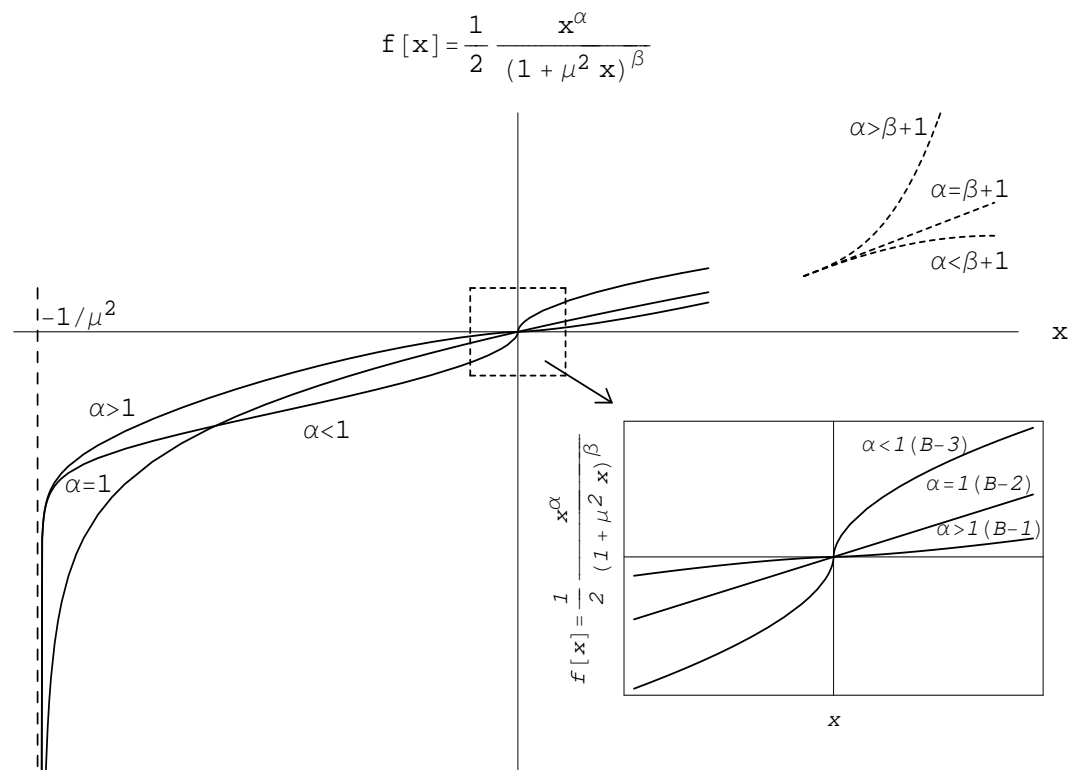

Fig. 6. Form of the lagrangian functions corresponding to models of the family (3.16) for three sets of values of the parameter $\alpha(\lesseqgtr 1)$. All lagrangians diverge at $X \rightarrow-1 / \mu^{2}$, which corresponds to the maximum value of the field strength. At the point $X \nsupseteq 0$, which determines the asymptotic behaviour of the solitons, the three sets of values of $\alpha$ give the three different behaviours: case B-1 $(\alpha>0)$, case B-2 $(\alpha=0)$ and case B-3 $(\alpha<0)$. The dashed lines show the behaviour of the lagrangian function at large positive values of $X$ for the admissible models corresponding to different relations among the parameters.

We also impose the condition $\alpha>\beta+1 / 2$, necessary to ensure the positivity of the energy density for any $X \in \Omega$. Moreover, the convergence of the integral of energy for the SSS solutions as $r \rightarrow \infty$ requires $\alpha<3 / 2$, as can be easily verified from the analysis of the field equation (2.4) and the integral of energy in this limit. Let us summarize in the following equations the restrictions imposed on the parameters of the models (3.16) in order to obtain admissible models with soliton solutions:

$$
\frac{1}{2}<\alpha \equiv \frac{\text { odd }}{\text { odd }}<\frac{3}{2} \quad ; \quad \beta \equiv \frac{\text { odd }}{\text { even }} \text { or irrational } \quad ; \quad 0<\beta<\alpha-\frac{1}{2}<1 .
$$

where the terms "odd" and "even" are implicitly understood to apply for natural numbers. The D'Alembert lagrangian is a limit member of this family obtained as $\alpha \rightarrow 1$ and $\beta \rightarrow 0$ or as $\alpha \rightarrow 1$ and $\mu \rightarrow 0$.

As results from the analysis of the A-2 cases, near the center the SSS solutions behave as

$$
\phi^{\prime}(r \rightarrow 0) \sim \frac{1}{\mu}-\lambda r^{\sigma},
$$

where the exponent $\sigma$ is given by

$$
1<\sigma=\frac{2}{1+\beta}<2,
$$

and $\lambda$ is a positive constant, which is the solution of the equation

is to show how models with such a kind of singularities can also lead to physically reasonable results. 


$$
2 \alpha \mu \lambda+\beta=\Lambda \mu^{(\alpha+\beta)}(2 \lambda)^{\beta+1} .
$$

As in the preceding examples, the energy of the soliton solutions can be explicitly obtained from (2.45). The final expression is

$$
\epsilon=\frac{4 \sqrt{2} \pi}{3} \frac{\Lambda^{3 / 2}}{\alpha^{1 / 2}}|\mu|^{\frac{2 \alpha-3}{2}} B\left(\frac{3-2 \alpha}{4}, \frac{\beta+3}{2}\right) F_{1}^{2}\left(\frac{1}{2}, \frac{3-2 \alpha}{4}, \frac{9-2(\alpha-\beta)}{4}, \frac{\alpha-\beta}{\alpha}\right),
$$

where $B(x, y)$ is again the Euler beta-function and $F_{1}^{2}(a, b, c, z)$ is the hypergeometric function defined as

$$
F_{1}^{2}=F(a, b, c, z)=\frac{1}{B(b, c-b)} \int_{0}^{1} t^{b-1}(1-t)^{c-b-1}(1-t z)^{-a} d t, \operatorname{Re}(b)>0, \operatorname{Re}(c)>0
$$

In figure 7 we have plotted the energy as a function of $\alpha$ with $\mu$ and $\beta$ as parameters. For a given value of $\mu$ the energy is rather insensitive to the parameter $\beta$. As $\alpha$ approaches the limit 3/2 the energy diverges for all values of $\mu$ and $\beta$. As a function of $\mu$ the energy decreases as the power $1 / \mu^{\left(\frac{3}{2}-\alpha\right)}$.

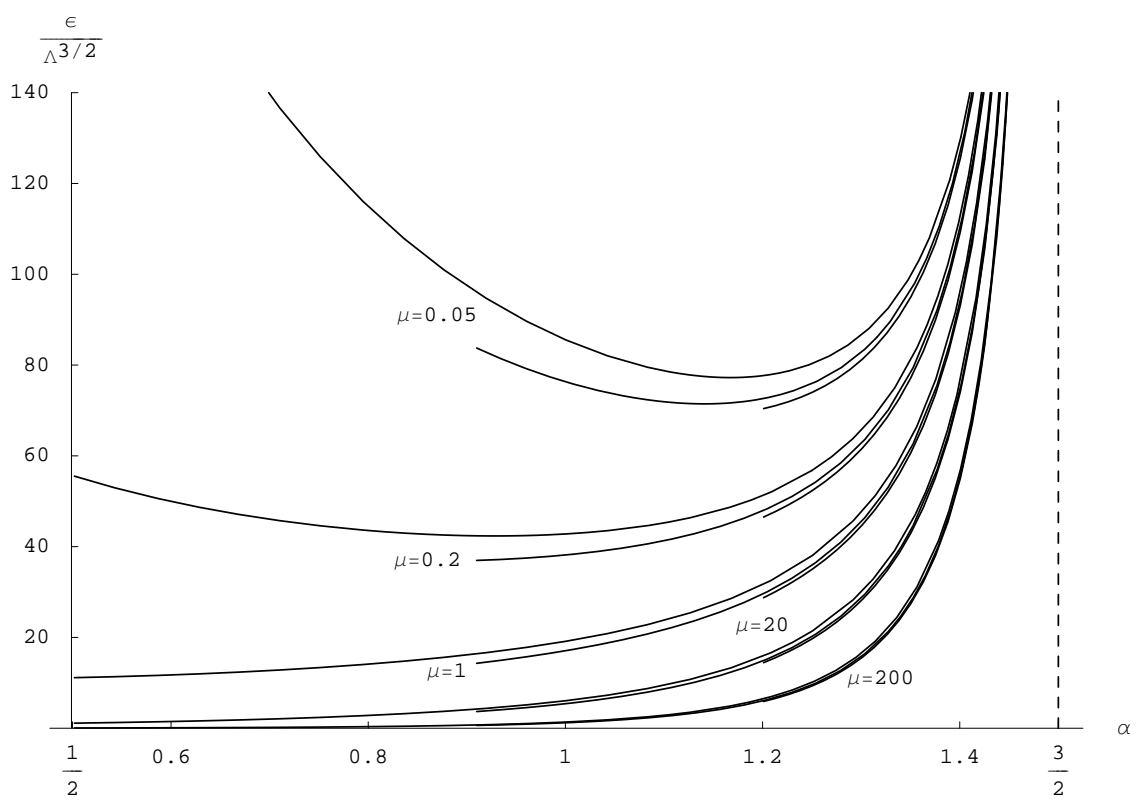

Fig. 7. Behaviour of the energy as a function $\alpha$ for five values of $\mu$ and three of $\beta(0.01,0.4,0.7)$. Note that the lower branches for each value of $\mu$ do not cover all the range $1 / 2<\alpha<3 / 2$ since the constraint $\alpha>\beta+1 / 2$ must be always fulfilled for admissibility.

\subsection{Short-ranged solutions}

As a fourth example let us look for a family of models whose SSS solutions are exponentially damped for large $r$ 18. In obtaining these models we shall proceed backwards, looking

\footnotetext{
${ }^{18}$ As already mentioned, these kinds of theories allow to describe short-range interactions through the exchange of self-coupled scalar fields. Their generalizations to the case of gauge fields, performed in section 5, lead to effective non-linear lagrangians
} 
for lagrangians whose associated field equations have prescribed SSS solutions. In this way we shall look for a family of lagrangian density functions of the form (2.1) whose associated SSS field solutions have the simple exponentially damped form 19

$$
\phi^{\prime}(r, \Lambda)=A \exp \left(-\sigma \frac{r^{n}}{\Lambda^{n / 2}}\right)
$$

where $A, \sigma$ and $n$ are positive constants determining the different models within this family 20 . The constant $\Lambda$ is the integration constant of the first-integral (2.4) of the field equation (whose solution is required to be (3.23)) and parameterizes all SSS solutions of a given model. It is explicitly introduced in (3.23) by implementing the scale law (2.7). These fields belong to the cases A-2 (finite field strength at the center) and B-3 (asymptotic damping faster than coulombian), and the corresponding models are class-2 field theories.

By eliminating $r$ between (3.23) and the first-integral (2.4) we obtain the form of the first derivative of the lagrangian density

$$
\dot{f}(X)=\frac{\Lambda}{r^{2} \phi^{\prime}(r)}=\frac{(2 \sigma)^{2 / n}}{\sqrt{-X} \ln ^{2 / n}\left(\frac{-A^{2}}{X}\right)},
$$

which holds in the interval $-A^{2}<X<0$ (where the SSS solution (3.23) is defined) and diverges at the boundaries. The lagrangian density in this interval is

$$
f(X)=2 \sigma^{2 / n} \int_{\sqrt{-X}}^{0} \frac{d y}{\ln ^{2 / n}(A / y)}=-2 A \sigma^{2 / n} \int_{\ln (A / \sqrt{-X})}^{\infty} \frac{e^{-z}}{z^{2 / n}} d z .
$$

As easily verified, $f(0)=0$ for any set of positive values of the parameters. In the lower boundary of the interval we have $f\left(X=-A^{2}\right)=-2 A \sigma^{2 / n} \Gamma\left(1-\frac{2}{n}\right)$ for $n>2$ (with $\Gamma(t)=$ $\int_{0}^{\infty} z^{t-1} e^{-z} d z, t>0$ being the usual Euler gamma function) and $f\left(X \rightarrow-A^{2}\right) \rightarrow-\infty$ for $n \leq 2$ (see figure 8). This expression of the lagrangian density could be continued to the region $X>0$ by matching (3.25) to any function satisfying the admissibility conditions there, but such continuations do not affect the structure of the solitons, which is completely determined by the part (3.25) of the lagrangian density 21 .

We calculate now the energy of these soliton solutions starting by convenience from Eq.(2.10) (although formula (2.45) would also work). After a partial integration we obtain

$$
\epsilon=\frac{4 \pi}{3}\left[-\left.r^{3} f\left(-\phi^{\prime 2}(r)\right)\right|_{0} ^{\infty}+\int_{0}^{\infty} r^{3} \frac{d f}{d r} d r\right] .
$$

which also describe short-range interactions and preserve the explicit gauge-invariance. From this point of view such models provide alternatives to the usual symmetry breaking mechanism in the description of weak interactions.

${ }^{19}$ More complex choices of exponentially damped SSS fields (as, for example, $\phi^{\prime}(r)=a(r) \exp \left(-\sigma \frac{r^{n}}{\Lambda^{n / 2}}\right)$, where $a(r)$ is assumed to be a bounded function) may be analyzed in a similar way, but in the present example the calculations can be performed in terms of elementary functions. With this choice the SSS field will be a soliton, but the method works also in obtaining models with exponentially-damped SSS solutions which are energy-divergent.

${ }^{20}$ The constant $A$ is a parameter of the model and not an integration constant of the solutions. It plays the role of the maximum field strength and is shared by all SSS solutions of a given model, but differs for the various models in the family.

${ }^{21}$ Nevertheless, the requirements of positivity of the energy and vanishing vacuum energy of the complete lagrangians are still necessary for the stability of the solitons (see section 6). 


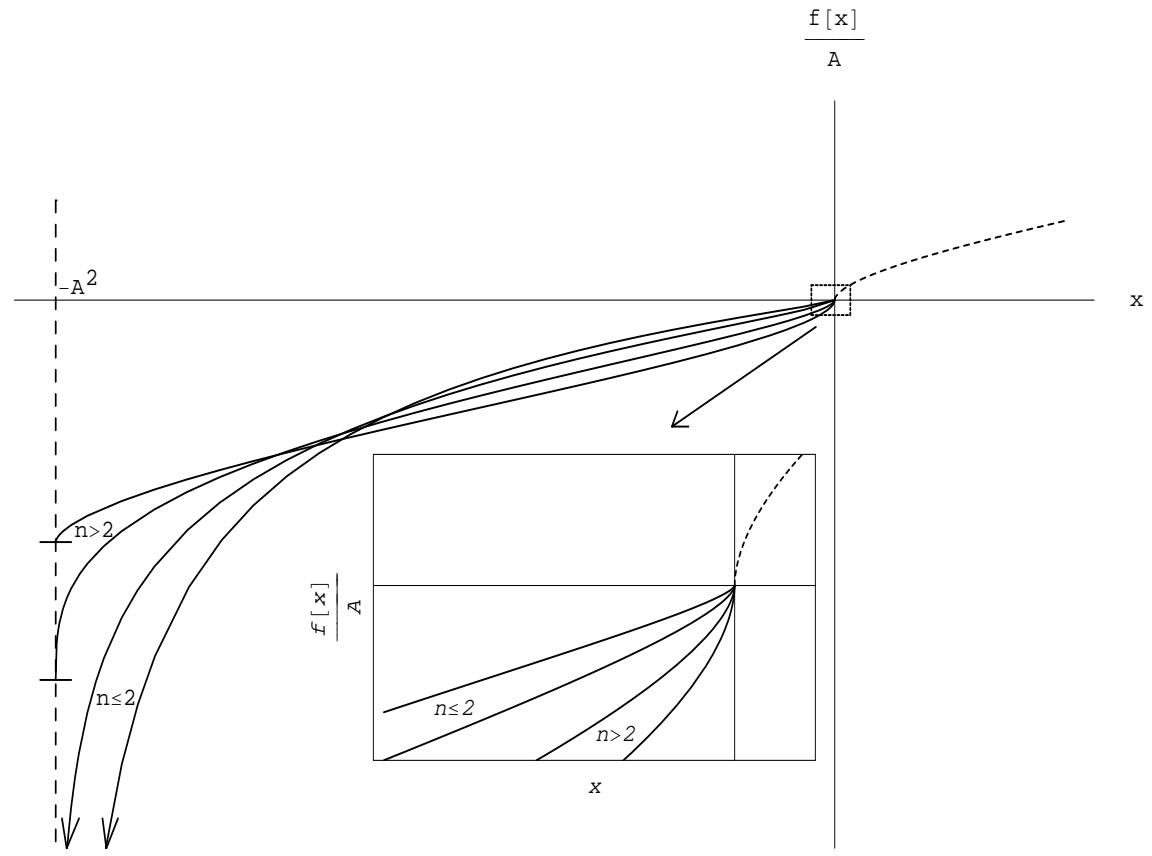

Fig. 8. Functional form of the lagrangian densities for the family (3.25), exhibiting short-ranged soliton solutions. The dashed line indicates possible continuations of the lagrangian density for $X>0$.

The integrated part in this equation can be shown to vanish for the prescribed solutions. In calculating the integral in the second term we use the first-integral field equation (2.4) and the expression of the field (3.23), which leads to

$$
\frac{d f}{d r}=-2 \dot{f}(X) \phi^{\prime}(r) \phi^{\prime \prime}(r)=\frac{-2 \Lambda}{r^{2}} \phi^{\prime \prime}(r)=2 \Lambda^{(2-n) / 2} A n \sigma r^{n-3} \exp \left(-\sigma \frac{r^{n}}{\Lambda^{n / 2}}\right),
$$

and the final expression for the energy of the solitons reads

$$
\epsilon=\frac{8 \pi A}{3 \sigma^{1 / n}} \Lambda^{3 / 2} \Gamma\left(\frac{1+n}{n}\right),
$$

which is proportional to the maximum amplitude of the field strength and decreases as the range of the field is reduced. In figure 9 we have plotted the energy of the unit maximumfield-strength as a function of $n$ for several values of the constant $\sigma$. As we see the energy diverges as $n \rightarrow 0$ and becomes less dependent on the constant $\sigma$ for large values of $n$, approaching asymptotically a fixed value $\frac{\epsilon}{A \Lambda^{3 / 2}}=\frac{8 \pi}{3}$.

\section{The multicomponent scalar field}

We shall extend the results of section 2 to the case of a set of scalar fields $\phi_{i}(x)(i=$ $1 \ldots N)$. In many cases the covariant lagrangians including $N$ scalar fields and their firstorder derivatives are constrained by conditions which allow to implement some internal symmetries. Such conditions manifest themselves in the structure of the manifold where the field takes its values. A well-known example is the non-linear sigma model where this manifold is a Riemann space implementing chiral symmetry [9], 39]. Here we shall restrict 


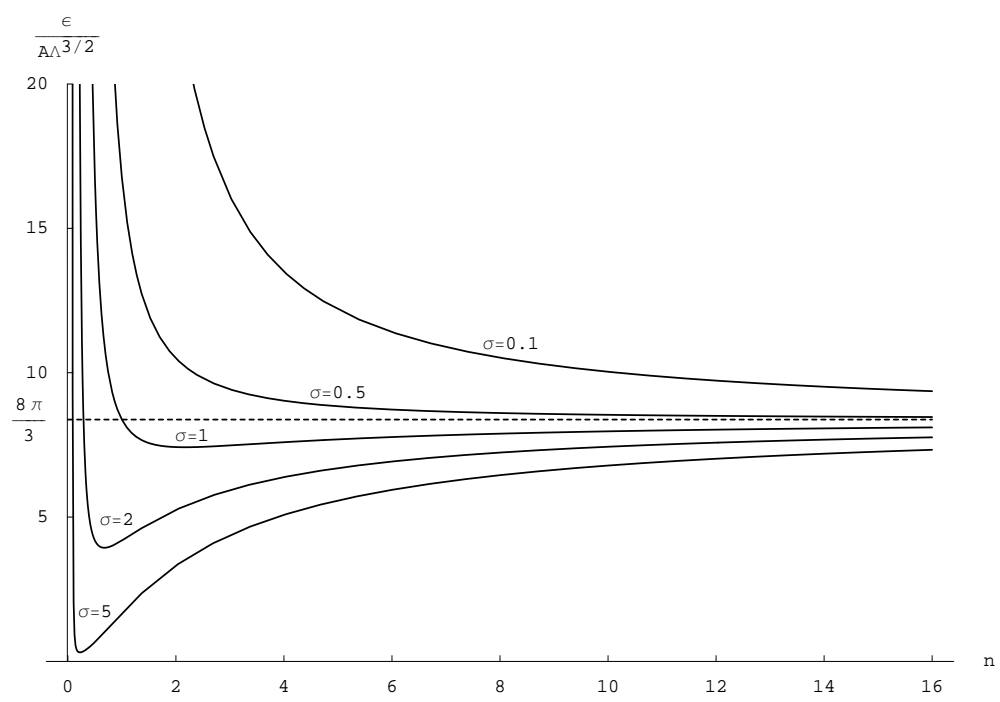

Fig. 9. Energy for short-ranged solitons (3.23) of unit maximum-field strength as a function of the exponent $n$, for several values of the parameter $\sigma$ (note that we have plotted $\frac{\epsilon}{A \Lambda^{3 / 2}}$ instead of $\frac{\epsilon}{\Lambda^{3 / 2}}$ as in the previous examples).

ourselves to the case where the field manifold is the $N$-dimensional Euclidean space and the $S O(N)$ invariant lagrangian density depends only on derivative terms

$$
L\left(\phi_{i}, \partial_{\mu} \phi_{i}\right)=f\left(\sum_{i=1}^{N} \partial_{\mu} \phi_{i} \partial^{\mu} \phi_{i}\right),
$$

where, as in section $2, f(X)$ is a given continuous, derivable $\left(C^{1}\right.$ for $\left.X<0\right)$ and monotonically increasing $\left(\frac{d f}{d X}>0, \forall X \neq 0 ; \frac{d f}{d X} \geq 0, X=0\right)$ function defined in a open and connected domain $(\Omega \subseteq \Re)$ which includes the vacuum $((X=0) \in \Omega)$. Besides the fact that these models are the natural generalizations of the scalar field theories studied so far, there is another motivation for their analysis. Indeed, as we shall see in the following sections, a class of soliton solutions arising in generalized gauge field theories of some compact semi-simple Lie groups of dimension $N$ reduce to multiscalar ( $N$ components) solitons of some of the models (4.1).

The field equations associated to the lagrangians (4.1) take the form of $N$ local conservation laws

$$
\partial_{\mu} J_{i}^{\mu}=0,
$$

where the conserved currents $J_{i}^{\mu}$ are

$$
J_{i}^{\mu}=\stackrel{\bullet}{f}(X) \partial^{\mu} \phi_{i},
$$

with $X=\sum_{i=1}^{N} \partial_{\alpha} \phi_{i} \cdot \partial^{\alpha} \phi_{i}$. The canonical energy-momentum tensor is

$$
T_{\mu \nu}=2 \dot{f}(X) \sum_{i=1}^{N} \partial_{\mu} \phi_{i} \partial_{\nu} \phi_{i}-f(X) \eta_{\mu \nu}
$$

and the corresponding energy density 


$$
\rho(x)=2 \dot{f}(X) \sum_{i=1}^{N}\left(\frac{\partial \phi_{i}}{\partial t}\right)^{2}-f(X),
$$

is positive definite under the same conditions established for the lagrangian function $f(X)$ of the one-component case.

For the SSS solutions $\phi_{i}(r)$, equations (4.2) have $N$ first-integral field equations of the form

$$
r^{2} \phi_{i}^{\prime} \dot{f}\left(-\sum_{j=1}^{N} \phi_{j}^{\prime 2}\right)=\Lambda_{i}
$$

where $\phi_{i}^{\prime}=d \phi_{i} / d r$ and $\Lambda_{i}$ are the integration constants. Now the signs of every component of the scalar field and of the corresponding integration constant are the same, but may differ for different components. In order to solve the system (4.6) let us introduce the functions $X_{i}(r)=-\phi_{i}^{\prime 2}(r)$, in such a way that $X(r)=\sum_{i=1}^{N} X_{i}(r)$. By squaring and adding Eqs.(4.6) we obtain

$$
r^{4} X \stackrel{\bullet}{f}^{2}(X)=-\sum_{i=1}^{N} \Lambda_{i}^{2},
$$

or, equivalently,

$$
r^{2} \sqrt{-X} \dot{f}(X)=\Lambda,
$$

where

$$
\Lambda=\sqrt{\sum_{i=1}^{N} \Lambda_{i}^{2}} .
$$

Equation (4.8) has the same form as the first-integral of the one-component scalar case (2.4). Consequently, if the function $f(X)$ is the same in both cases, we can associate to any SSS solution of the one-component case, of the form $\phi^{\prime}(r, \Lambda)$, a set of sequences of $N$ functions which are SSS solutions of the multicomponent scalar equations. Such functions take the form

$$
\phi_{i}^{\prime}\left(r, \Lambda_{j}\right)=\frac{\Lambda_{i}}{\Lambda} \phi^{\prime}(r, \Lambda)
$$

and, owing to Eq.(4.9), there is a one-to-one correspondence between such sequences and the points of the sphere of radius $\Lambda$ in the $N$-dimensional Euclidean space $\left(\Re^{N}\right)$. Obviously, this is a straightforward consequence of the invariance of the lagrangian (4.1) under rotations in the internal space. The constants $\Lambda_{i}$ can now be identified as the "source point-charges" associated to the different components of the SSS field, namely

$$
\Lambda_{i}=\frac{1}{4 \pi} \int d^{3} x \vec{\nabla} \cdot\left(\stackrel{\bullet}{f}(X) \vec{\nabla} \phi_{i}\right) .
$$

The field potentials obtained by integration of (4.10) read

$$
\phi_{i}\left(r, \Lambda_{j}\right)=\frac{\Lambda_{i}}{\Lambda} \phi(r, \Lambda)+\Delta_{i}
$$


where $\Delta_{i}$ are integration constants and $\Lambda$ is the mean-square scalar charge (4.9).

If we consider now the energy associated to these SSS solutions we find from (4.5)

$$
\epsilon=-4 \pi \int_{0}^{\infty} r^{2} f\left(\sum_{i=1}^{N} X_{i}(r)\right) d r=-4 \pi \int_{0}^{\infty} r^{2} f(X(r)) d r .
$$

This is the energy of the one-component SSS solution corresponding to the integration constant (4.9). Thus the set of SSS solutions of the multicomponent scalar field associated to the points of the sphere of radius $\Lambda$ in $\Re^{N}$ is degenerate in energy. Moreover, the search for conditions to be imposed on $f(X)$ for the existence of finite-energy SSS solutions (as well as the admissibility constraints) in the multicomponent case reduces to the analysis of section 2 for the one-component case.

Concerning the conditions for stability of the solutions (4.12), the analysis of the onecomponent case can be straightforwardly generalized to the present situation (see subsection 6.3. for details). The final conclusion is that the multicomponent soliton solutions of admissible models are also linearly stable against charge-preserving perturbations.

\section{Gauge fields}

We shall now extend the analysis developed in the previous sections to generalized gauge field theories of compact semi-simple Lie groups. To start with, we study the simpler case of generalized ( $U(1)$-invariant) electromagnetic fields before proceeding further to the case of non-abelian gauge fields.

\subsection{Abelian case}

We define lagrangian densities for generalized electromagnetic fields defined as arbitrary functions of the two quadratic field invariants, built from the Maxwell tensor and its dual. Following the conventions of Ref. [42, these tensors are defined as

$$
\begin{aligned}
& F_{\mu \nu}=\partial_{\mu} A_{\nu}-\partial_{\nu} A_{\mu} \\
& F_{\mu \nu}^{*}=\frac{1}{2} \varepsilon_{\mu \nu \alpha \beta} F^{\alpha \beta},
\end{aligned}
$$

where $\varepsilon^{0123}=-\varepsilon_{0123}=1$. The electric and magnetic fields are defined as $E^{i}=-F^{0 i}$ and $H^{i}=-\frac{1}{2} \varepsilon^{i j k} F_{j k}$ while the quadratic invariants $X$ and $Y$ are

$$
\begin{aligned}
X & =-\frac{1}{2} F_{\mu \nu} F^{\mu \nu}=\vec{E}^{2}-\vec{H}^{2} \\
Y & =-\frac{1}{2} F_{\mu \nu} F^{* \mu \nu}=2 \vec{E} \cdot \vec{H} .
\end{aligned}
$$

We define the general form of the lagrangian density as

$$
L=\varphi(X, Y),
$$

where $\varphi$ is a given continuous and derivable function on its domain of definition $(\Omega)$ of the $X-Y$ plane $\left(\Re^{2}\right)$. As in the scalar case we assume $\Omega$ to be open and connected and including 
the vacuum $((X=0, Y=0) \in \Omega)$. As a minimal extension of the assumptions of section 2 concerning the regularity properties of the scalar lagrangian functions, which is necessary for future purposes (see Eq.(15.20) below), we shall assume $\varphi(X, Y)$ to be of class $C^{1}$ on the line $(X>0, Y=0) \cap \Omega$ and $\partial \varphi / \partial X$ to be strictly positive there. By generalizing the definitions of section 2 we shall call "class-1 field theories" the models defined and regular everywhere $\left(\Omega \equiv \Re^{2}\right)$ and "class-2 field theories" those with $\Omega \subset \Re^{2}$. We also require $\varphi(X, Y)$ to be symmetric in the second argument, in order to implement parity invariance

$$
\varphi(X, Y)=\varphi(X,-Y) .
$$

This implies that the odd partial derivatives of $\varphi(X, Y)$ with respect to $Y$ must vanish on $Y=0$. The Maxwell lagrangian density corresponds to $\varphi(X, Y)=\frac{X}{8 \pi}$, while the Born-Infeld electrodynamics is given by the lagrangian density

$$
L_{B-I}=\varphi_{B-I}(X, Y)=\frac{1-\sqrt{1-\mu^{2} X-\frac{\mu^{4}}{4} Y^{2}}}{4 \pi \mu^{2}},
$$

where $\frac{1}{\mu}$ is the maximum field strength, attained at the center of the solution. The lagrangian (5.5) reduces to the Maxwell one in the limit $\mu \rightarrow 0$.

The symmetric (gauge-invariant) energy-momentum tensor obtained from the lagrangian density (5.3) takes the form

$$
T_{\mu \nu}^{s}=2\left(\frac{\partial \varphi}{\partial X} F_{\mu \alpha} F_{\nu}^{\alpha}+\frac{\partial \varphi}{\partial Y} F_{\mu \alpha} F_{\nu}^{* \alpha}\right)-\varphi \eta_{\mu \nu}=2 \frac{\partial \varphi}{\partial X} F_{\mu \alpha} F_{\nu}^{\alpha}+\left(Y \frac{\partial \varphi}{\partial Y}-\varphi\right) \eta_{\mu \nu}
$$

and the associated energy density is

$$
\rho^{s}=T_{00}^{s}=2 \frac{\partial \varphi}{\partial X} \vec{E}^{2}+2 \frac{\partial \varphi}{\partial Y} \vec{E} \cdot \vec{H}-\varphi(X, Y)=2 X \frac{\partial \varphi}{\partial X}-\varphi(X, Y)+Y \frac{\partial \varphi}{\partial Y}+2 \frac{\partial \varphi}{\partial X} \vec{H}^{2} .
$$

We assume the symmetric energy-momentum tensor (5.6) to give the correct space-time energy density distribution. Let us analyze the conditions for the positivity of the energy density of any field configuration. The inspection of Eq.(5.7), together with the requirement of vanishing of the vacuum energy, lead to the set of necessary conditions

$$
\varphi(0,0)=0 \quad ; \quad \varphi(X, 0)<0 \quad \forall(X<0, Y=0) \in \Omega \quad ; \quad \frac{\partial \varphi}{\partial X}>0 \quad \forall(X, Y) \in \Omega,
$$

to be satisfied by the lagrangian densities. However, it is possible to obtain a minimal set of necessary and sufficient conditions of admissibility for a satisfactory energetic behaviour. Solving Eqs.(5.2) for the fields we obtain

$$
\begin{aligned}
& E^{2}=\frac{1}{2}\left(\sqrt{X^{2}+\frac{Y^{2}}{\cos ^{2}(\vartheta)}}+X\right) \geq \frac{1}{2}\left(\sqrt{X^{2}+Y^{2}}+X\right) \\
& H^{2}=\frac{1}{2}\left(\sqrt{X^{2}+\frac{Y^{2}}{\cos ^{2}(\vartheta)}}-X\right) \geq \frac{1}{2}\left(\sqrt{X^{2}+Y^{2}}-X\right),
\end{aligned}
$$

where $\vartheta$ is the angle between $\vec{E}$ and $\vec{H}$. From these expressions the energy density can be written as 


$$
\rho^{s}=\frac{\partial \varphi}{\partial X}\left(\sqrt{X^{2}+\frac{Y^{2}}{\cos ^{2}(\vartheta)}}+X\right)+Y \frac{\partial \varphi}{\partial Y}-\varphi(X, Y) .
$$

Consequently, the requirement of the positive definite character of the energy leads to the minimal necessary and sufficient condition

$$
\rho^{s} \geq\left(\sqrt{X^{2}+Y^{2}}+X\right) \frac{\partial \varphi}{\partial X}+Y \frac{\partial \varphi}{\partial Y}-\varphi(X, Y) \geq 0
$$

to be satisfied in the entire domain of definition $(\Omega)$. Generalizing the criteria of section 2 , we only regard as "admissible" those models whose lagrangian densities satisfy the condition (5.11), aside from the vanishing of the vacuum energy and the regularity and parityinvariance conditions stated above 22. Consequently, the admissible lagrangians must be solutions of the first-order linear inhomogeneous partial differential equation

$$
\left(\sqrt{X^{2}+Y^{2}}+X\right) \frac{\partial \varphi}{\partial X}+Y \frac{\partial \varphi}{\partial Y}-\varphi(X, Y)=\Psi(X, Y)
$$

where $\Psi(X, Y)$ is any function being positive definite in $\Omega$ and vanishing in vacuum $(\Psi(0,0)=$ $0)$. Such solutions must also satisfy the regularity and parity-invariance requirements, as supplementary conditions.

Let us now consider the trace of the symmetric energy-momentum tensor

$$
T^{s}=4\left[\frac{\partial \varphi}{\partial X}\left(\vec{E}^{2}-\vec{H}^{2}\right)+2 \frac{\partial \varphi}{\partial Y} \vec{E} \cdot \vec{H}-\varphi(X, Y)\right]=4\left[X \frac{\partial \varphi}{\partial X}+Y \frac{\partial \varphi}{\partial Y}-\varphi(X, Y)\right]
$$

From the last expression we see that the sub-class of models with traceless symmetric energymomentum tensors is given by the lagrangian densities $\varphi(X, Y)$ which are solutions of the first-order linear homogeneous partial differential equation

$$
X \frac{\partial \varphi}{\partial X}+Y \frac{\partial \varphi}{\partial Y}-\varphi(X, Y)=0
$$

The general solution of this equation is the family of all conic surfaces in the $(X, Y, \varphi)$-space having the origin as a vertex. Clearly the set of planes of the form $\varphi=a X+b Y$ ( $a$ and $b$ being constants) are particular solutions of this equation (which violate parity invariance if $b \neq 0)$. The simple case $b=0$ with $a=\frac{1}{8 \pi}$ corresponds to the Maxwell theory.

The field equations obtained from the lagrangian (5.3) are

$$
\partial_{\mu}\left(\frac{\partial \varphi}{\partial X} F^{\mu \nu}+\frac{\partial \varphi}{\partial Y} F^{* \mu \nu}\right)=0
$$

to which we add the Bianchi identities

$$
\partial_{\mu} F^{* \mu \nu}=0 .
$$

In terms of the fields these equations read

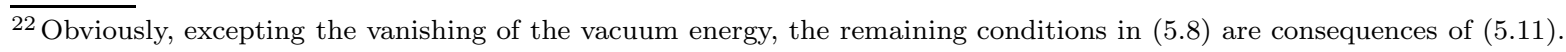




$$
\begin{aligned}
& \vec{\nabla} \cdot\left(\frac{\partial \varphi}{\partial X} \vec{E}+\frac{\partial \varphi}{\partial Y} \vec{H}\right)=0 \\
& -\frac{\partial}{\partial t}\left(\frac{\partial \varphi}{\partial X} \vec{E}+\frac{\partial \varphi}{\partial Y} \vec{H}\right)+\vec{\nabla} \times\left(\frac{\partial \varphi}{\partial X} \vec{H}-\frac{\partial \varphi}{\partial Y} \vec{E}\right)=0
\end{aligned}
$$

and

$$
\begin{aligned}
\vec{\nabla} \times \vec{E} & =-\frac{\partial \vec{H}}{\partial t} \\
\vec{\nabla} \cdot \vec{H} & =0 .
\end{aligned}
$$

For electrostatic fields we have $Y=0$ and in the ESS case these equations can be written in terms of the electrostatic potential $A^{0}(r)\left(\vec{A}=0, \vec{E}(r)=-\vec{\nabla} A^{0}(r)\right)$ in such a way that the first group of equations in (5.17) leads to the first-integral 23

$$
r^{2} \frac{d A^{0}}{d r} \frac{\partial \varphi}{\partial X}(X, Y=0)=q
$$

where now $X=\left(\frac{d A^{0}}{d r}\right)^{2}$ and $q$ is an integration constant. Using the identification $\phi(r, \Lambda) \equiv$ $A^{0}(r, q=\Lambda)$ this equation coincides with the first-integral (2.4) for the SSS solutions of a scalar field model with a lagrangian density defined by

$$
L_{\text {scalar }}=f\left(\partial_{\mu} \phi \cdot \partial^{\mu} \phi\right) \equiv f(X)=-\varphi(-X, Y=0),
$$

which leads to

$$
\dot{f}(X)=\frac{\partial \varphi}{\partial X}(-X, Y=0) .
$$

Conversely we can associate to each scalar model defined by a lagrangian density $f(X)$, a family of electromagnetic field models defined by lagrangian densities $\varphi(X, Y)$ satisfying Eq.(5.20) as well as the admissibility (5.11) and stability constraints (see Eq.(5.30) below). The ESS field solutions of all electromagnetic generalizations $(|E(\vec{r}, q)|)$ have the same form, as functions of $r$, as the SSS field solutions $\left(\phi^{\prime}(r, \Lambda)\right)$ of the original scalar model, $q$ and $\Lambda$ being the integration constants, which should be identified as the electric and scalar point-like charges associated to the solution, respectively. Indeed, in the generalized electromagnetic case the definition of the electric charge associated to a given field is

$$
\frac{1}{4 \pi} \int d^{3} r \vec{\nabla} \cdot\left(\frac{\partial \varphi}{\partial X} \vec{E}+\frac{\partial \varphi}{\partial Y} \vec{H}\right)
$$

which now is conserved as a consequence of the field equations. Substituting in this equation the ESS field coming from the solution of (5.19) we obtain $q$ as the value of its total electric charge

$$
\frac{1}{4 \pi} \vec{\nabla} \cdot\left[\frac{\partial \varphi}{\partial X} \vec{E}\right]=q \delta_{3}(\vec{r}) .
$$

$\overline{{ }^{23}}$ The remaining three equations in (5.17) are identically satisfied for arbitrary electrostatic fields, owing to Eq. (5.4). On the other hand, the set of equations (5.18) are trivially satisfied by the ESS solutions. 
As pointed out in Ref. 3] for the Born-Infeld model this charge can be interpreted as a source point-like charge at the center of the ESS solution or, alternatively, as a continuous charge-density distribution associated with the field and given by

$$
\frac{1}{4 \pi} \frac{\partial \varphi}{\partial X}(X=0, Y=0) \vec{\nabla} \cdot \vec{E}
$$

This interpretation, as already discussed for scalar models, requires the function $r^{2} E(r)$ to vanish at the origin (this condition is always fulfilled for the finite-energy ESS solutions) and the field $E(r)$ to be asymptotically coulombian (B-2 case models).

In calculating the total energy of these electrostatic central fields from the energy density (5.7) we are lead to

$$
\begin{aligned}
\epsilon_{e}(q) & =8 \pi \int_{0}^{\infty} r^{2} \frac{\partial \varphi}{\partial X}\left[X=\vec{E}^{2}(r, q), Y=0\right] \vec{E}^{2}(r, q) d r- \\
& -4 \pi \int_{0}^{\infty} r^{2} \varphi\left[X=\vec{E}^{2}(r, q), Y=0\right] d r
\end{aligned}
$$

(the index e stands for electric field). The energy associated with the corresponding SSS scalar field solutions, obtained from Eqs.(2.10) and (5.20) reads

$$
\epsilon_{s}(\Lambda)=4 \pi \int_{0}^{\infty} r^{2} \varphi\left[\vec{E}^{2}(r, \Lambda), 0\right] d r
$$

(the index s stands for scalar field). If the total energy (5.26) associated to the scalar field is finite, so is $\epsilon_{e}(q)$. Indeed, using Eq.(5.19) this energy becomes

$$
\epsilon_{e}(q)=8 \pi q\left[A^{0}(\infty, q)-A^{0}(0, q)\right]-\epsilon_{s}(q),
$$

which, owing to (2.12), is related with the scalar soliton energy through

$$
\epsilon_{e}(q)=2 \epsilon_{s}(q)
$$

and must be also finite. Thus the energy of a ESS solution is twofold the energy of the corresponding SSS soliton when the integration constants take the same value $(\Lambda=q)$. Equivalently, Eq.(2.10), which gives the scaling of energies, leads to the following relation between the integration constants (the electric $(q)$ and the scalar $(\Lambda)$ charges) of an electrostatic soliton and the associated scalar soliton of equal-energy

$$
q=(2)^{2 / 3} \Lambda \text {. }
$$

We then conclude that the ESS solutions for the families of electromagnetic models which generalize (through Eq.(5.20) ) the different classes of scalar models with soliton solutions, have the same functional forms as the corresponding SSS scalar solutions and are also of finite-energy. Moreover, the classification of the admissible models with soliton solutions in the scalar case according to the central and asymptotic behaviours of the fields, immediately induces, through Eq.(5.20), a similar classification of the finite-energy ESS solutions in the electromagnetic case. 
Let us point out an immediate consequence of this analysis (which is a corollary of the non-existence theorems established in Refs. [20,, [43]): there are not ESS soliton solutions for admissible generalized electromagnetic field theories with traceless energy-momentum tensor. Indeed, as mentioned above the lagrangian densities of such theories (see Eq.(5.13) ) are given by conic surfaces in the $(X, Y, \varphi)$ space and the associated scalar field lagrangian densities $f(X)=-\varphi(-X, Y=0)$ are straight lines in the $(X, f)$ plane for $X<0$. Consequently the associated SSS solutions are coulombian in form as well as energy-divergent, and so are the ESS solutions of these generalized electromagnetic models.

Although finite-energy SSS solutions of admissible scalar models are always linearly stable against charge-preserving perturbations, this is not so for the finite-energy ESS solutions of admissible generalized electromagnetic theories. Indeed, the analysis of the linear stability of the electrostatic solitons leads to a generalization of the criteria obtained in the scalar case (see section 6 for details). As a result of this analysis, the electrostatic finite-energy central field solutions of admissible generalized electromagnetic field models, with their lagrangian densities $\varphi(X, Y)$ satisfying the supplementary condition

$$
\frac{\partial \varphi}{\partial X}-2 X \frac{\partial^{2} \varphi}{\partial Y^{2}}>0
$$

in the entire domain of existence of the ESS solutions in the plane $(X, Y=0)$, can be shown to be local minima of the energy functional against small charge-preserving perturbations. Consequently, admissibility, finite-energy condition of the ESS solutions aside from Eq.(5.30) are necessary and sufficient conditions for linear static stability. Moreover, the linear analysis of dynamics of the small perturbations of the soliton solutions performed for the scalar models can be generalized to the associated families of electromagnetic models which satisfy (5.30) (see section 6).

Finally, the conditions for univoque and everywhere defined ESS solutions are straightforwardly deduced from those of the scalar case, aside from Eq.(5.20).

\subsection{Non-abelian case}

The results obtained for generalized electromagnetic fields can be extended to non-abelian generalized gauge field theories of compact semi-simple Lie groups of dimension $N$. As usually, in this case the tensor field strength components in the algebra and their duals are defined from the gauge fields $A_{a \mu}$ and the structure constants $C_{a b c}$ as

$$
\begin{aligned}
& F_{a \mu \nu}=\partial_{\mu} A_{a \nu}-\partial_{\nu} A_{a \mu}-g \sum_{b c} C_{a b c} A_{b \mu} A_{c \nu} \\
& F_{a \mu \nu}^{*}=\frac{1}{2} \varepsilon_{\mu \nu \alpha \beta} F_{a}^{\alpha \beta},
\end{aligned}
$$

whose components define the fields $\vec{E}_{a}, \vec{H}_{a}$ in the usual form. In order to introduce the lagrangian densities governing the generalized dynamics of these fields we must define pertinent field invariants. However there is now an ambiguity in the calculation of the traces over the group indices, leading to different possibilities in the definition of these invariants. Although at this regard different prescriptions have been introduced, mainly in the context 
of string theory, where B-I-like actions arise as a low-energy effective field limit [16], [44, here we shall restrict our analysis to the case of actions built from the two simplest first-order field invariants, defined from the ordinary prescription for the calculation of the traces as

$$
\begin{aligned}
X & =-\frac{1}{2} \sum_{a}\left(F_{a \mu \nu} F_{a}^{\mu \nu}\right)=\sum_{a}\left(\vec{E}_{a}^{2}-\vec{H}_{a}^{2}\right) \\
Y & =-\frac{1}{2} \sum_{a}\left(F_{a \mu \nu} F_{a}^{* \mu \nu}\right)=2 \sum_{a}\left(\vec{E}_{a} \cdot \vec{H}_{a}\right),
\end{aligned}
$$

where $1 \leq a \leq N$. The generalized lagrangian density is now assumed to be a given function $\varphi(X, Y)$ which (again for parity invariance) must be symmetric in the second argument $(\varphi(X, Y)=\varphi(X,-Y))$ and satisfy the same admissibility constraints of definition, continuity and derivability (as well as the distinction between class-1 and class-2 field theories) as in the electromagnetic case.

The associated field equations read now

$$
\sum_{c} D_{a c \mu}\left[\frac{\partial \varphi}{\partial X} F_{c}^{\mu \nu}+\frac{\partial \varphi}{\partial Y} F_{c}^{* \mu \nu}\right]=0
$$

where $D_{a c \mu} \equiv \delta_{a c} \partial_{\mu}-g \sum_{b} C_{a b c} A_{b \mu}$ is the gauge-covariant derivative.

The symmetric energy-momentum tensor is

$$
T_{\mu \nu}^{s}=2 \sum_{a} F_{a \mu \alpha}\left(\frac{\partial \varphi}{\partial X} F_{a \nu}^{\alpha}+\frac{\partial \varphi}{\partial Y} F_{a \nu}^{* \alpha}\right)-\varphi \eta_{\mu \nu},
$$

and the energy density takes the form

$$
\rho^{s}=T_{00}^{s}=2 \frac{\partial \varphi}{\partial X} \sum_{a} \vec{E}_{a}^{2}+2 \frac{\partial \varphi}{\partial Y} \sum_{a} \vec{E}_{a} \cdot \vec{H}_{a}-\varphi(X, Y) .
$$

The admissibility conditions to be imposed on the lagrangian density, for the energy functional to be positive definite and vanishing in vacuum, take the same form as in the electromagnetic case (see Eqs.(5.8) and (5.11)). Also the trace of the energy-momentum tensor has the same expression (see the last member of Eq.(15.13)), and vanishes under the same conditions (5.14). The subclass of models with non-vanishing trace energy-momentum tensor breaks the scale invariance and thus circumvent the non-existence theorems [20, [43] allowing for soliton-like solutions.

Let us consider the ESS solutions of these models. We consider fields of the form

$$
\vec{E}_{a}(\vec{r})=-\vec{\nabla}\left(A_{a}^{0}(r)\right)=-A_{a}^{\prime 0}(r) \frac{\vec{r}}{r} \quad ; \quad \vec{H}_{a}=0,
$$

where the functions $A_{a}^{0}(r)$ are the time-like components of the gauge potential in the Lorentz gauge $\left(\vec{A}_{a}=0\right)$ and $A_{a}^{\prime 0}=\frac{d A_{a}^{0}}{d r}$. When replaced in the field equations (5.33) we are lead to

$$
\begin{aligned}
& \vec{\nabla} \cdot\left(\frac{\partial \varphi}{\partial X} \vec{E}_{a}\right)=-\vec{\nabla} \cdot\left(\frac{\partial \varphi}{\partial X} \vec{\nabla} A_{a}^{0}(r)\right)=-\vec{\nabla} \cdot\left(\frac{\partial \varphi}{\partial X} A_{a}^{\prime 0}(r) \frac{\vec{r}}{r}\right)=0 \\
& \frac{\partial \varphi}{\partial X} \sum_{b c} C_{a b c} A_{b}^{0}(r) \vec{E}_{c}=-\frac{\partial \varphi}{\partial X} \sum_{b c} C_{a b c} A_{b}^{0}(r) \vec{\nabla} A_{c}^{0}(r)=
\end{aligned}
$$




$$
=-\frac{\partial \varphi}{\partial X} \sum_{b c} C_{a b c} A_{b}^{0}(r) A_{c}^{\prime 0}(r) \frac{\vec{r}}{r}=0,
$$

where $X=\sum_{a} \vec{E}_{a}^{2}$. The first group of equations has a set of first-integrals of the form

$$
r^{2} \frac{\partial \varphi}{\partial X} A_{a}^{\prime 0}(r)=Q_{a}
$$

where the $Q_{a}$ are integration constants which will be identified below as "source color charges". With the identification $\phi_{a}(r) \equiv A_{a}^{0}(r)$ and $\Lambda_{a} \equiv Q_{a}$, these equations coincide with the field equations (4.6) for a multicomponent SSS scalar field theory whose lagrangian density is given by

$$
L=f\left(\sum_{a} \partial_{\mu} \phi_{a} \cdot \partial^{\mu} \phi_{a}\right) \equiv f(X)=-\varphi(-X, Y=0) .
$$

Thus the solutions of equations (5.38) are obtained from Eq.(4.10) as

$$
\left|\vec{E}_{a}\left(r, Q_{b}\right)\right|=A_{a}^{\prime 0}\left(r, Q_{b}\right)=\frac{Q_{a}}{Q} \phi^{\prime}(r, Q) \quad ; \quad \vec{H}_{a}=0,
$$

where $Q=\sqrt{\sum_{a} Q_{a}^{2}}$ is the mean-square color charge and $\phi^{\prime}(r, Q)$ is the SSS solution of the associated one-component scalar model defined by a lagrangian density of the form (5.39). These equations can be integrated once, leading to

$$
A_{a}^{0}\left(r, Q_{b}\right)=\frac{Q_{a}}{Q} \phi(r, Q)+\chi_{a}
$$

where $\chi_{a}$ are integration constants. These functions must also satisfy the second set of equations (5.38). Owing to the antisymmetry of the structure constants these equations lead to the supplementary restriction

$$
\chi_{a}=\frac{Q_{a}}{Q} \chi
$$

and the final solution of (5.38) is

$$
A_{a}^{0}\left(r, Q_{b}\right)=\frac{Q_{a}}{Q}(\phi(r, Q)+\chi),
$$

where $\chi$ is an arbitrary constant, being now the same for all components of the potential. In terms of the fields the final solution is still given by Eq.(5.40). The integration constants $Q_{a}$ in this solution must be interpreted as source point-like color charges associated with the different components of the gauge field. Indeed, color charges are defined in general as

$$
Q_{a}=\frac{1}{4 \pi} \int d_{3} \vec{r} \vec{\nabla} \cdot\left(\frac{\partial \varphi}{\partial X} \vec{E}_{a}+\frac{\partial \varphi}{\partial Y} \vec{H}_{a}\right),
$$

which, owing to the field equations (5.33), include now external source charges and charges carried by the field itself. The latter ones come from the integration of the term

$$
g \sum_{b c} C_{a b c} A_{b \mu}\left[\frac{\partial \varphi}{\partial X} F_{c}^{\mu \nu}+\frac{\partial \varphi}{\partial Y} F_{c}^{* \mu \nu}\right]
$$


which vanishes for the ESS solutions. On the other hand the former are associated to Dirac distributions of weight $4 \pi Q_{a}$, as can be easily seen from the substitution of Eqs.(5.38) in the first set of Eqs.(5.38).

The calculation of the energy associated to these solutions proceeds in the same way as in the electromagnetic case. The integration of Eq.(5.35) gives

$$
\begin{aligned}
\epsilon_{g f}\left(\Lambda_{a}\right) & =8 \pi \int_{0}^{\infty} r^{2} \frac{\partial \varphi}{\partial X}\left[X=\sum_{b} \vec{E}_{b}^{2}\left(r, \Lambda_{a}\right), Y=0\right] \sum_{b} \vec{E}_{b}^{2}\left(r, \Lambda_{a}\right) d r- \\
& -4 \pi \int_{0}^{\infty} r^{2} \varphi\left[X=\sum_{b} \vec{E}_{b}^{2}\left(r, \Lambda_{a}\right), Y=0\right] d r
\end{aligned}
$$

where the index $\mathbf{g f}$ stands for gauge field. From this expression it is now straightforward to show that, as in the corresponding multiscalar case, this energy is finite if the energy of the associated scalar solitons is finite, and depends on the charges only through the constant $Q$, having the same kind of degeneration on spheres of radius $Q$ in the $N$-dimensional colorcharge space. The relation between the finite energies of the solitons with equal mean-square charges in the gauge models and in the associated multiscalar models is given by the same equation (5.28) relating the finite energies in the cases of the abelian models and their associated scalar models.

Concerning the stability of the gauge field solitons we shall show in the next section that the finite-energy ESS solutions of admissible generalized non-abelian models are linearly stable if (and only if) the lagrangian density functions satisfy the same criterion (5.30) obtained in the abelian case.

Let us summarize the main conclusions of this section: The set of generalized gauge field theories of compact semi-simple Lie groups, whose lagrangian densities are functions $\varphi(X, Y)$ of the field invariants (5.32), satisfying the admissibility conditions and the stability criterion (5.30), and supporting finite-energy ESS non-topological soliton solutions, can be split in equivalence classes. Two models belong to the same class if their respective lagrangian densities satisfy the condition $\varphi_{1}(X, 0)=\varphi_{2}(X, 0)$. The forms of the ESS soliton solutions and their energies coincide for all models belonging to the same class. There is a one-toone correspondence, given by Eq.(5.20), between the set of these classes and the set of admissible scalar field models defined by Eq.(2.1) and supporting finite-energy SSS nontopological solitons. The forms and energies of the gauge solitons are obtained from those of the corresponding scalar solitons through Eqs.(5.40), (5.41) and (5.28). The analysis and classification of scalar solitons performed in section 2 can be immediately generalized to gauge solitons through this correspondence. Furthermore, the explicit examples supporting scalar solitons, introduced in section 3, can also be extended to the gauge field case simply by including the $Y$ invariant in such a way that the admissibility (5.11) and stability (5.30) constraints be fulfilled by the extended models.

\section{Stability}

Let us now analyze the stability of the finite-energy solutions of the different models introduced so far. We make a distinction between "strong" stability, defined as the ability of 
a soliton to maintain its identity under any perturbation or in closed many-soliton configurations [4],45], and "weak" stability, identified with the usual linear stability under small perturbations. Rigorous analysis of stability in the strong sense has been performed for a few field theories in one-space dimension which exhibit conserved discrete topological charges associated with the soliton solutions. In three-space dimensions similar topological conservation laws are responsible for the stability of the 't Hooft-Polyakov monopole solution 5] or the chiral soliton solution of Deser et al. [9]. But satisfactory general methods for the analysis of interactions between non-topological solitons and strong external fields in three space dimensions are still lacking and only numerical analysis of the evolution of the solutions can give some insight on this issue for most models. In our context, a tentative approach to this question has been developed by Chernitsky for the Born-Infeld model [46]. It is based on the use of the discontinuity of the field strength at the center of static B-I solitons as a marker of the presence and location of the dynamic soliton evolving in interaction with strong external fields, or in many-soliton configurations. Since all SSS soliton solutions of the models considered here exhibit similar central field singularities, this procedure might be extended to these cases, but such an extension lies beyond the scope of the present work.

In the case of interactions between solitons and weak external fields (or for widely separated soliton configurations) linear stability ensures the identity preservation of the solitons and becomes a basic condition for the consistency of the low-energy analysis. The results of this analysis may be interpreted in terms of particle-field (or particle-particle) force laws and describe the radiative behaviour in these processes [46].

\subsection{Static stability of scalar solitons.}

We shall begin with the study of the static linear stability for the soliton solutions of the scalar models of section 2, by analyzing the behaviour of their energy, which must be a minimum against appropriate small perturbations 24 . We shall show that, for these models, the conditions of admissibility guarantee this kind of stability for all finite-energy SSS solutions. We start with the SSS potential $\phi(r)$ and introduce a set of small static perturbations $\delta \phi(\vec{r})$, finite and regular (as well as their first order spatial derivatives) everywhere. We also require the perturbation to leave unchanged the scalar charge associated to the solution 25. To first order in the perturbations the modification of this scalar charge, obtained from Eq.(2.5), reads

$$
\Delta \Lambda=\frac{1}{4 \pi} \int d_{3} \vec{r} \vec{\nabla} \cdot\left[\stackrel{\bullet}{f}\left(X_{0}\right)(\vec{\nabla} \delta \phi)-2 \ddot{f}\left(X_{0}\right)(\vec{\nabla} \phi \cdot \vec{\nabla} \delta \phi) \vec{\nabla} \phi\right]=0
$$

where now $X_{0}=-(\vec{\nabla}(\phi))^{2}=-\phi^{\prime 2}(r)$. The condition $\Delta \Lambda=0$ imposes restrictions on the behaviour of the admissible perturbations at $r=0$ and as $r \rightarrow \infty$. In particular, $\delta \phi(\vec{r})$ must satisfy

\footnotetext{
${ }^{24}$ As emphasized in Ref. [47 this criterion is a sufficient condition for stability, but is not a necessary one. Here we shall not consider the problem of stability of solutions which do not correspond to minima of the energy, a complicated task which deserves a study in itself.

${ }^{25}$ This condition is essential for the energy of the soliton to be a minimum. Indeed, without such a condition the perturbation does not necessarily lead to an increase of the energy of the soliton (as can be easily seen by differentiating Eq. 2.10) with respect to small variations of $\Lambda$ ) and the perturbed soliton might evolve towards less energetic states.
} 


$$
\lim _{r \rightarrow \infty} \frac{\delta \phi(\vec{r})}{\phi(\infty)-\phi(r)}=0 .
$$

In this manner the perturbed fields remain inside the space of functions defined by the prescribed boundary conditions (on $S_{\infty}$ in this case) which determine uniquely the solution associated to a given value of the charge. At the center of the soliton $\delta \phi(\vec{r})$ must be regular (see Eq.(6.27) and the analysis of the dynamic stability below).

The first-order perturbation of the energy, calculated by expanding (2.10) becomes

$$
\begin{aligned}
\Delta_{1} \epsilon & =2 \int d_{3} \vec{r} \dot{f}\left(X_{0}\right) \vec{\nabla}(\phi) \cdot \vec{\nabla}(\delta \phi)= \\
& =2 \int d_{3} \vec{r} \vec{\nabla} \cdot\left(\dot{f}\left(X_{0}\right) \delta \phi \vec{\nabla}(\phi)\right)-2 \int d_{3} \vec{r} \delta \phi \vec{\nabla} \cdot\left(\dot{f}\left(X_{0}\right) \vec{\nabla}(\phi)\right),
\end{aligned}
$$

where a partial integration has been performed. Owing to Eq.(2.4) and the assumed asymptotic behaviour of the perturbation $\delta \phi(\vec{r})$, the two integrals in the last equation converge and cancel each other so that the first variation of the energy vanishes. This is the necessary condition for the energy of the soliton to be an extremum. The second variation reads

$$
\Delta_{2} \epsilon=\int d_{3} \vec{r} \dot{f}\left(X_{0}\right)(\vec{\nabla} \delta \phi)^{2}-2 \int d_{3} \vec{r} \ddot{f}\left(X_{0}\right)(\vec{\nabla} \phi \cdot \vec{\nabla} \delta \phi)^{2},
$$

where, owing to the boundary behaviours of the perturbation and the SSS field itself, both integrals are also convergent. From the arbitrariness of $\delta \phi$, the positivity of $\dot{f}(X)$ and the minimum condition of the energy $\Delta_{2} \epsilon>0$, we see that static stability is reached if the requirement

$$
\ddot{f}(X)<0,
$$

is fulfilled in all the range of values of $X=X_{0}=-\phi^{\prime 2}(r)$ covered by the solution. However, if we rewrite Eq.(6.4) as

$$
\begin{aligned}
\Delta_{2} \epsilon & =\int d_{3} \vec{r}\left[\dot{f}\left(X_{0}\right)+2 X_{0} \ddot{f}\left(X_{0}\right)\right]\left(\frac{\partial \delta \phi}{\partial r}\right)^{2}+ \\
& +\int d_{3} \vec{r} \dot{f}\left(X_{0}\right)\left[\frac{1}{r^{2}}\left(\frac{\partial \delta \phi}{\partial \theta}\right)^{2}+\frac{1}{r^{2} \cos ^{2}(\theta)}\left(\frac{\partial \delta \phi}{\partial \varphi}\right)^{2}\right],
\end{aligned}
$$

we are lead to the less restrictive static stability criterion 26

$$
\dot{f}\left(X_{0}\right)+2 X_{0} \ddot{f}\left(X_{0}\right) \geq 0,
$$

which is a necessary and sufficient condition for linear stability, as opposed to Eq.(6.5) which is only a sufficient one. This criterion is always fulfilled for admissible models with finiteenergy SSS solutions. Indeed, by deriving the first-integral equation (2.4) with respect to $r$ we obtain

$$
\dot{f}\left(X_{0}\right)-2 \phi^{\prime 2}(r) \ddot{f}\left(X_{0}\right)=-\frac{2 \Lambda}{r^{3} \phi^{\prime \prime}(r)},
$$

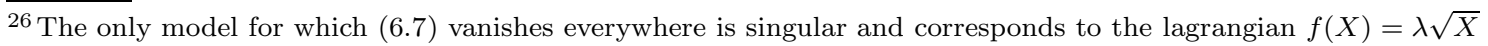


which, owing to the monotonicity of $\phi^{\prime}(r)$, is positive in all the range of values of $X_{0}$ covered by the solution. We conclude that the finite-energy SSS solutions of admissible scalar models are statically stable.

Against perturbations which modify the charge the solitons are unstable, but these instabilities are blocked if charge conservation is implicit in the model (as in the case of generalized gauge field theories considered below) or if it is a consequence of the nature of the external sources.

\subsection{Dynamic stability of scalar solitons.}

Let us now consider the dynamic stability of the SSS solutions. The initial perturbation defines the following Cauchy conditions

$$
\Phi(\vec{r}, t=0)=\phi(r)+\delta \phi(\vec{r}) \quad ; \quad \frac{\partial \Phi}{\partial t}(\vec{r}, t=0)=0
$$

for a dynamical problem determining the temporal evolution of the perturbed field $\Phi(\vec{r}, t)$, which is governed by the hyperbolic field equations (2.2). At the first order, the evolution of the perturbation

$$
\delta \phi(\vec{r}, t)=\Phi(\vec{r}, t)-\phi(r),
$$

is given by the linearized scalar field equation

$$
\frac{\partial}{\partial t}\left(\stackrel{\bullet}{f}\left(X_{0}\right) \frac{\partial \delta \phi}{\partial t}\right)-\vec{\nabla} \cdot\left[\stackrel{\bullet}{f}\left(X_{0}\right) \vec{\nabla}(\delta \phi)-2 \ddot{f}\left(X_{0}\right)(\vec{\nabla} \phi \cdot \vec{\nabla} \delta \phi) \vec{\nabla} \phi\right]=0 .
$$

This is the Euler-Lagrange equation associated with the lagrangian density

$$
L=\frac{1}{2}\left[\dot{f}\left(X_{0}\right) \partial_{\mu} \delta \phi \cdot \partial^{\mu} \delta \phi-2 \stackrel{\bullet}{f}\left(X_{0}\right)(\vec{\nabla} \phi \cdot \vec{\nabla} \delta \phi)^{2}\right],
$$

which is defined everywhere. Equation (6.11) has the form of a local conservation law for a charge density $\eta=\dot{f}\left(X_{0}\right) \frac{\partial \delta \phi}{\partial t}$ which, in integral form, becomes

$$
\frac{d}{d t} \int d_{3} \vec{r} \dot{f}\left(X_{0}\right) \frac{\partial \delta \phi}{\partial t}=\int d_{3} \vec{r} \vec{\nabla} \cdot\left[\stackrel{f}{f}\left(X_{0}\right) \vec{\nabla}(\delta \phi)-2 \stackrel{\bullet}{f}\left(X_{0}\right)(\vec{\nabla} \phi \cdot \vec{\nabla} \delta \phi) \vec{\nabla} \phi\right]=0 .
$$

The r.h.s. of this equation is proportional to the first-order perturbation of the scalar charge of the soliton (see Eq.(6.1) ) which, consequently, remains conserved as time evolves. Moreover, for solutions satisfying the initial conditions (6.9) the quantity

$$
\int d_{3} \vec{r} \dot{f}\left(X_{0}\right) \delta \phi(\vec{r}, t)
$$

remains constant in time.

Equation (6.11), together with the conditions (6.9) and (6.2), outline a spectral problem to which we can apply standard methods. We look for solutions separating time and spatial variables under the form

$$
\delta \phi(\vec{r}, t, \Gamma)=T(t, \Gamma) \psi(\vec{r}, \Gamma),
$$


where $\Gamma$ is the separation constant and the eigenfunction $\psi(\vec{r}, \Gamma)$ is assumed to satisfy the boundary condition (6.2). Replacing this expression in (6.11) and using the second of the initial conditions (6.9) we are lead to

$$
T(t, \Gamma)=\cos (\sqrt{\Gamma} t)
$$

and

$$
\Gamma \dot{f}\left(X_{0}\right) \psi=-\vec{\nabla} \cdot\left[\dot{f}\left(X_{0}\right) \vec{\nabla}(\psi)-2 \stackrel{f}{f}\left(X_{0}\right) \phi^{\prime 2}\left(\frac{\vec{r}}{r} \cdot \vec{\nabla} \psi\right) \frac{\vec{r}}{r}\right] .
$$

The sign of the eigenvalue $\Gamma$ is crucial for stability. Multiplying this equation by $\psi(\vec{r}, \Gamma)$ and integrating over all space we are lead (after an integration by parts of the right-hand-side) to

$$
\Gamma \int d_{3} \vec{r} \dot{f}\left(X_{0}\right) \psi^{2}=\int d_{3} \vec{r}\left[\dot{f}\left(X_{0}\right)(\vec{\nabla} \psi)^{2}-2 \ddot{f}\left(X_{0}\right) \phi^{\prime 2}\left(\frac{\partial \psi}{\partial r}\right)^{2}\right] .
$$

Owing to Eq. (6.8), together with the admissibility and boundary conditions, both the r.h.s. of this equation and the coefficient of $\Gamma$ are finite and positive. Then so is for $\Gamma$, and the evolution is oscillatory and bounded in time 27. Moreover, if we consider two different eigenvalues $\left(\Gamma_{i}, i=(1,2)\right)$ and their associated eigenfunctions $\left(\psi_{i}=\psi\left(\vec{r}, \Gamma_{i}\right), i=(1,2)\right)$ equation (6.17) leads to

$$
\left(\Gamma_{2}-\Gamma_{1}\right) \int d_{3} \vec{r} \dot{f}\left(X_{0}\right) \psi_{1} \psi_{2}=\int d_{3} \vec{r}\left(\psi_{2} \vec{\nabla} \cdot \vec{\Sigma}_{1}-\psi_{1} \vec{\nabla} \cdot \vec{\Sigma}_{2}\right),
$$

where

$$
\vec{\Sigma}_{i}=\stackrel{\bullet}{f}\left(X_{0}\right) \vec{\nabla} \psi_{i}-2 \stackrel{\bullet}{f}\left(X_{0}\right)\left(\vec{\nabla} \phi \cdot \vec{\nabla} \psi_{i}\right) \vec{\nabla} \phi .
$$

After a partial integration and making use of the boundary conditions we see that the right-hand-side of (6.19) vanishes and thus we are lead to the orthogonality relation

$$
\int d_{3} \vec{r} \dot{f}\left(X_{0}\right) \psi_{1} \psi_{2}=0
$$

These results outline a Sturm-Liouville problem for each admissible scalar model of the form (2.1) supporting finite-energy SSS solutions and lead to the following conclusions [48]: i) The analysis of the dynamics of the small oscillations around these solutions leads in all cases to discrete spectra of eigenvalues $\Gamma_{i}$, ii) The associated eigenfunctions are orthogonal and finite-norm with respect to the scalar product

$$
<\psi_{i}, \psi_{j}>=\int d_{3} \vec{r} \dot{f}\left(X_{0}\right) \psi_{i}(\vec{r}) \psi_{j}(\vec{r})=\frac{\Delta_{2} \epsilon_{i}}{\Gamma_{i}} \delta_{i j}
$$

defined with the kernel $\dot{f}\left(X_{0}\right)>0$. Such functions generate a complete Hilbert space in which any perturbation can be expanded.

\footnotetext{
${ }^{27}$ Note that the r.h.s. of 6.18$)$ coincides with the second variation of the energy associated to the eigenfunction $\psi(\vec{r}, \Gamma)$ and has the same sign as $\Gamma$. This establishes a strict correspondence between static and dynamic stabilities of the SSS solutions.
} 
On the other hand, we can now separate the spatial eigenfunctions in radial and angular coordinates as

$$
\psi(r, \vartheta, \varphi, \Gamma, l)=R(r, \Gamma, l) Y_{l}(\vartheta, \varphi),
$$

where the angular components are the usual spherical harmonics satisfying

$$
\sin \vartheta \frac{\partial}{\partial \vartheta}\left(\sin \vartheta \frac{\partial Y_{l}}{\partial \vartheta}\right)+\frac{\partial^{2} Y_{l}}{\partial \varphi^{2}}+l(l+1) \sin ^{2} \vartheta Y_{l}=0 .
$$

The radial components obey to the equation

$$
\frac{d}{d r}\left(\frac{1}{r \phi^{\prime \prime}} \frac{d R}{d r}\right)+\frac{l(l+1)-\Gamma r^{2}}{2 r^{2} \phi^{\prime}} R=0
$$

which is obtained from (6.17), (6.23) and using the first-integral (2.4). These equations have the standard Sturm-Liouville form [48]

$$
\begin{aligned}
& L y+\lambda \mu(x) y=0 \\
& L y=\frac{d}{d x}\left[k(x) \frac{d y}{d x}\right]-q(x) y \quad \text { with } k(x)>0, \mu(x)>0 .
\end{aligned}
$$

The asymptotic behaviour of $R(r)$ is obtained from the asymptotic form of the admissible solitons $\left(\phi^{\prime}(r \rightarrow \infty) \sim 1 / r^{p} ; p>1\right)$ through

$$
\frac{d^{2} R}{d r^{2}}+\frac{p}{r} \frac{d R}{d r}-p \frac{\left(l(l+1)-\Gamma r^{2}\right)}{2 r^{2}} R=0,
$$

which is a Lommel equation and can be solved in terms of Bessel functions [49]. For large $r$ we can assume for the solution the asymptotic form

$$
R(r \rightarrow \infty) \sim \frac{\varrho(r)}{r^{q}}
$$

where $\varrho(r)$ is a bounded function and, owing to the boundary condition (6.2), $q$ is restricted to be $q>p>1$. By neglecting the higher-order terms in $1 / r$ in the resulting equation for $\rho$ we are lead to

$$
\varrho^{\prime \prime}+\frac{p \Gamma}{2} \varrho=0 .
$$

Owing to the positivity of $\Gamma$ the solution of this equation is oscillatory and the asymptotic behaviour of the eigenfunctions is given by 28

$$
R(r \rightarrow \infty) \sim \frac{\cos \left(\sqrt{\frac{p \Gamma}{2}} r+\chi\right)}{r^{q}},
$$

where $\chi$ is a constant phase. This asymptotic form of the eigenfunctions makes the integral of the second variation of the energy (6.6) to converge in the $r \rightarrow \infty$ limit.

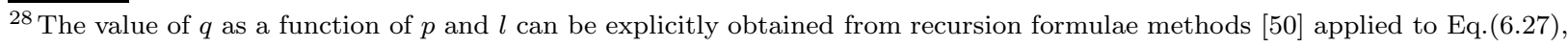
which also allow for the approximate determination of the eigenvalue spectrum of $\Gamma$. 
To determine the behaviour of $R(r)$ around the center of the soliton we must consider separately cases A-1 and A-2. Let us assume in both cases a form

$$
R(r \rightarrow 0) \sim \alpha-\beta r^{q} .
$$

In case A-1 $\left(\phi^{\prime}(r \rightarrow 0) \sim 1 / r^{p}, 0<p<1\right)$ equation (6.25) becomes

$$
2 \beta q \frac{p+q-1}{p} r^{q}+\left(l(l+1)-\Gamma r^{2}\right)\left(\alpha-\beta r^{q}\right) \approx 0 .
$$

For the first surface spherical harmonic $(l=0)$ we are lead to $[50$ ]

$$
\alpha=0 ; q=1-p,
$$

or

$$
\frac{\beta}{\alpha}=\frac{\Gamma p}{4(p+1)} \quad ; \quad q=2
$$

For $l \neq 0$ we obtain

$$
\alpha=0 \quad ; \quad q=\frac{1-p+\sqrt{(1-p)^{2}+2 l(l+1) p}}{2} .
$$

In case A-2 $\left(\phi^{\prime}(r \rightarrow 0) \sim a-b r^{\sigma}, \sigma>0\right)$ equation (6.25) becomes

$$
2 \beta q \frac{q-\sigma-1}{b \sigma} r^{q-\sigma}+\frac{l(l+1)-\Gamma r^{2}}{a}\left(\alpha-\beta r^{q}\right) \approx 0 .
$$

If $l=0$ we must have

$$
\frac{\beta}{\alpha}=\frac{b}{2 a} \frac{\Gamma \sigma}{\sigma+2} ; q=\sigma+2
$$

or

$$
\alpha=0 ; q=\sigma+1
$$

For $l \neq 0$

$$
\frac{\beta}{\alpha}=\frac{b}{2 a} l(l+1) ; q=\sigma,
$$

or

$$
\alpha=0 ; q=\sigma+1 .
$$

In all these cases the integral of the second order variation of the energy (6.6) can be shown to converge in the limit $r \rightarrow 0$.

\subsection{Stability of multicomponent scalar solitons.}

The analysis of stability in the scalar case can be extended to the multicomponent scalar fields. Now we have $N$ integration constants $\Lambda_{i}$ and a degeneration in energy of the SSS solutions on the sphere of radius $\Lambda=\sqrt{\sum_{i=1}^{N} \Lambda_{i}^{2}}$ in $\Re^{N}$. Obviously the variations of the 
energy vanish for perturbations which remain inside this sphere obtained by modifying the constants $\Lambda_{i}$ in equation (4.10) in such a way that the "total mean-square charge" $\Lambda$ remains unchanged. The asymptotic boundary conditions satisfied by the fields $\phi_{i}^{\prime}\left(r, \Lambda_{j}\right)$ (obtained from the asymptotic behaviour of the associated one-component scalar field solution $\phi^{\prime}(r, \Lambda)$ through Eq.(4.10) ) are modified by these perturbations and the associated charges (defined from Eq.(4.11) as $\Lambda_{i}$ ) are modified. Consequently, charge conservation condition blocks such perturbations and prevents a soliton from evolving spontaneously towards another equalenergy configuration in the sphere.

For general perturbations $\delta \phi_{i}(\vec{r})$ the first-order modifications of the scalar charges take the form

$$
\Delta \Lambda_{i}=\frac{1}{4 \pi} \int d_{3} \vec{r} \vec{\nabla} \cdot\left[\stackrel{f}{f}\left(X_{0}\right)\left(\vec{\nabla} \delta \phi_{i}\right)-2 \stackrel{\bullet}{f}\left(X_{0}\right) \sum_{j=1}^{N}\left(\vec{\nabla} \phi_{j} \cdot \vec{\nabla} \delta \phi_{j}\right) \vec{\nabla} \phi_{i},\right],
$$

with $X=-\sum_{i=1}^{N} \phi_{i}^{\prime 2}$. The requirement of charge conservation $\left(\Delta \Lambda_{i}=0\right)$ imposes boundary conditions on the perturbing fields which, as in the one-component case, must vanish asymptotically faster than the SSS fields themselves.

The first-order variation of the energy functional is obtained from the integral of Eq.(4.5) and reads

$$
\Delta_{1} \epsilon=2 \sum_{i=1}^{N} \frac{\Lambda_{i}}{\Lambda}\left(\int d_{3} \vec{r} \nabla \cdot\left[\stackrel{\bullet}{f}\left(X_{0}\right) \delta \phi_{i} \vec{\nabla} \phi\right]-\int d_{3} \vec{r} \delta \phi_{i} \vec{\nabla} \cdot\left[\stackrel{\bullet}{f}\left(X_{0}\right) \vec{\nabla}(\phi)\right]\right),
$$

where Eq.(4.12) has been used and a partial integration has been performed. Each term of this sum has the form of Eq. (6.4) and vanishes because of the same reasons. Thus the first variation of the energy vanishes, which is an extremum condition. The second variation of the energy functional takes the form

$$
\Delta_{2} \epsilon=\int d_{3} \vec{r}\left[\dot{f}\left(X_{0}\right) \sum_{i=1}^{N}\left(\vec{\nabla} \delta \phi_{i}\right)^{2}-2 \phi^{\prime 2} \stackrel{\bullet}{f}\left(X_{0}\right)\left(\sum_{i=1}^{N} \frac{\Lambda_{i}}{\Lambda} \frac{\partial \delta \phi_{i}}{\partial r}\right)^{2}\right],
$$

and can be written as

$$
\begin{aligned}
\Delta_{2} \epsilon & =\int d_{3} \vec{r}\left[\stackrel{\bullet}{f}\left(X_{0}\right)+2 X_{0} \ddot{f}\left(X_{0}\right) \cos ^{2}(\Omega)\right] \sum_{i=1}^{N}\left(\frac{\partial \delta \phi_{i}}{\partial r}\right)^{2}+ \\
& +\int d_{3} \vec{r} \dot{f}\left(X_{0}\right) \sum_{i=1}^{N}\left[\frac{1}{r^{2}}\left(\frac{\partial \delta \phi_{i}}{\partial \theta}\right)^{2}+\frac{1}{r^{2} \cos ^{2}(\theta)}\left(\frac{\partial \delta \phi_{i}}{\partial \varphi}\right)^{2}\right],
\end{aligned}
$$

in terms of the angle $\Omega(\vec{r})$ in the internal space between the vector formed by the radial derivatives of the components of the perturbing fields and the direction $\frac{\Lambda_{i}}{\Lambda}$ defined by the SSS solution. The second integral in this equation is always positive. If $\stackrel{\bullet}{f}\left(X_{0}\right)$ is negative the first integral is also positive. Otherwise we have

$$
\dot{f}\left(X_{0}\right)+2 X_{0} \ddot{f}\left(X_{0}\right) \cos ^{2}(\Omega) \geq \dot{f}\left(X_{0}\right)+2 X_{0} \ddot{f}\left(X_{0}\right) \geq 0,
$$

and, owing to Eq.(6.8), this integral is always positive for admissible many-components scalar models with finite-energy SSS solutions. Consequently, all these solutions are statically 
stable. Moreover, the analysis of the dynamical evolution of small perturbations performed for scalar solitons can be straightforwardly generalized to this multicomponent case. Such an analysis proves the dynamical stability of these solitons.

\subsection{Static stability of generalized electromagnetic solitons}

Similar analysis of stability can be performed for generalized abelian gauge fields. We consider a finite-energy ESS solution 29 of the field equations (5.15) $\left(\vec{E}_{0}(r), \vec{H}_{0}=0\right)$ and introduce a small perturbing field $\left(\vec{E}_{1}(\vec{r}), \vec{H}_{1}(\vec{r})\right)$ which does not modify the total electric charge of the soliton. The first-order modification of the charge density is obtained by perturbing the first of the field equations (5.17), which leads to

$$
\vec{\nabla} \cdot \vec{\sigma}=0
$$

where

$$
\vec{\sigma}=\frac{\partial \varphi}{\partial X_{0}} \vec{E}_{1}+2 \frac{\partial^{2} \varphi}{\partial X_{0}^{2}}\left(\vec{E}_{0} \cdot \vec{E}_{1}\right) \vec{E}_{0}
$$

and the index 0 in the derivatives means that they are calculated for the unperturbed solution (note that, owing to the parity invariance, the odd partial derivatives of $\varphi$ with respect to $Y$ vanish in $Y=0$ ). From the integration of (6.46) we see that $\vec{\sigma}$ must vanish asymptotically faster than $r^{-2}$. Then, the regular perturbing field $\left|\vec{E}_{1}(\vec{r})\right|$ must be damped faster than $E_{0}(r)$ itself. This boundary condition is similar to the one introduced for scalar models but, owing to the electric charge conservation implicit in the field equations, the physical meaning becomes here more transparent.

Let us now consider the variations of the energy functional under such charge-preserving perturbations. The first-order variation is obtained from the integration of Eq.(5.7) and reads

$$
\Delta_{1} \epsilon=-2 \int d_{3} \vec{r} \vec{\nabla} \cdot\left[A^{0} \vec{\sigma}\right]+2 \int d_{3} \vec{r} A^{0} \vec{\nabla} \cdot \vec{\sigma},
$$

where we have introduced the time-like component of the four-vector potential for the solution $\left(\vec{E}_{0}=-\vec{\nabla} A^{0}, \vec{A}=0\right)$. This expression vanishes due to the boundary conditions and the linearized field equation (6.46). This is an extremum condition.

In calculating the second variation of the energy let us expand the first of the field equations (5.17) to the second-order. We are lead to

$$
\vec{\nabla} \cdot(\vec{\sigma}+\vec{\eta})=0
$$

where now the term

$$
\begin{aligned}
\vec{\eta} & =2 \frac{\partial^{2} \varphi}{\partial X_{0}^{2}}\left(\vec{E}_{0} \cdot \vec{E}_{1}\right) \vec{E}_{1}+\frac{\partial^{2} \varphi}{\partial X_{0}^{2}}\left(\vec{E}_{1}^{2}-\vec{H}_{1}^{2}\right) \vec{E}_{0}+2 \frac{\partial^{3} \varphi}{\partial X_{0}^{3}}\left(\vec{E}_{0} \cdot \vec{E}_{1}\right)^{2} \vec{E}_{0}+ \\
& +2 \frac{\partial^{3} \varphi}{\partial X_{0} \partial Y_{0}^{2}}\left(\vec{E}_{0} \cdot \vec{H}_{1}\right)^{2} \vec{E}_{0}+2 \frac{\partial^{2} \varphi}{\partial Y_{0}^{2}}\left(\vec{E}_{0} \cdot \vec{H}_{1}\right) \vec{H}_{1}
\end{aligned}
$$

\footnotetext{
${ }^{29}$ To avoid difficulties related to the gauge determination we work directly with the fields. When the use of the potentials becomes necessary in some step of the calculation we will fix the gauge through appropriate conditions.
} 
includes the second-order corrections. Using this equation the second variation of the energy, obtained from the integration of Eq.(5.7), becomes

$$
\begin{aligned}
\Delta_{2} \epsilon & =\int d_{3} \vec{r}\left[\frac{\partial \varphi}{\partial X_{0}} \vec{E}_{1}^{2}+2 \frac{\partial^{2} \varphi}{\partial X_{0}^{2}}\left(\vec{E}_{0} \cdot \vec{E}_{1}\right)^{2}\right]+\int d_{3} \vec{r}\left[\frac{\partial \varphi}{\partial X_{0}} \vec{H}_{1}^{2}-2 \frac{\partial^{2} \varphi}{\partial Y_{0}^{2}}\left(\vec{E}_{0} \cdot \vec{H}_{1}\right)^{2}\right]- \\
& -2 \int d_{3} \vec{r} \vec{\nabla} \cdot\left[A^{0} \vec{\eta}\right] .
\end{aligned}
$$

The last integral in the r.h.s. of this equation vanishes, owing to the boundary conditions. The first term is positive if $\frac{\partial^{2} \varphi}{\partial X_{0}^{2}} \geq 0$; on the other hand, if $\frac{\partial^{2} \varphi}{\partial X_{0}^{2}}<0$ the integrand of this term can be written as

$$
\left(\vec{E}_{1}\right)^{2}\left(\frac{\partial \varphi}{\partial X_{0}}+2 \frac{\partial^{2} \varphi}{\partial X_{0}^{2}}\left(\vec{E}_{0}^{2} \cos ^{2}(\theta)\right)\right) \geq\left(\vec{E}_{1}\right)^{2}\left(\frac{\partial \varphi}{\partial X_{0}}+2 \frac{\partial^{2} \varphi}{\partial X_{0}^{2}} \vec{E}_{0}^{2}\right)
$$

where $\theta$ is the angle between $\vec{E}_{0}$ and $\vec{E}_{1}$. By deriving Eq.(5.19) with respect to $r$ and taking into account the monotonicity of $E_{0}(r)$ we see that the first term in (6.51) is always positive. Concerning the second term of (6.51) it is positive if $\frac{\partial^{2} \varphi}{\partial Y_{0}^{2}} \leq 0$ while if $\frac{\partial^{2} \varphi}{\partial Y_{0}^{2}}>0$ we can write its integrand as

$$
\left(\vec{H}_{1}\right)^{2}\left(\frac{\partial \varphi}{\partial X_{0}}-2 \frac{\partial^{2} \varphi}{\partial Y_{0}^{2}}\left(\vec{E}_{0}^{2} \cos ^{2}(\theta)\right)\right) \geq\left(\vec{H}_{1}\right)^{2}\left(\frac{\partial \varphi}{\partial X_{0}}-2 \frac{\partial^{2} \varphi}{\partial Y_{0}^{2}}\left(\vec{E}_{0}^{2}\right)\right) .
$$

From the arbitrariness of the perturbing fields, the positivity of this term and, finally, the positivity of the second variation of the energy requires the condition

$$
\frac{\partial \varphi}{\partial X} \geq 2 X \frac{\partial^{2} \varphi}{\partial Y^{2}}
$$

to be fulfilled in the range of values of $X(Y=0)$ where the ESS solutions are defined. This is a necessary and sufficient condition of static stability to be satisfied by the lagrangian densities of admissible models supporting finite-energy ESS solutions. This stability criterion goes beyond the widely used Derrick's necessary conditions [10].

Let us check, using this criterium, the linear stability of the electrostatic finite-energy solutions of the B-I model. From Eqs. (5.5) and (6.54) we immediately obtain

$$
\frac{\partial \varphi}{\partial X_{0}}-2 X_{0} \frac{\partial^{2} \varphi}{\partial Y_{0}^{2}}=\frac{1}{8 \pi}\left(1-\mu^{2} X_{0}\right)^{1 / 2}>0
$$

and the stability condition (6.54) is fulfilled since the ESS field is bounded everywhere $\left(X_{0}<1 / \mu^{2}\right)$.

\subsection{Dynamic stability of generalized electromagnetic solitons}

Let us now analyze the dynamical evolution of the small perturbations of the ESS solitons. The system of linearized field equations, obtained by expanding (5.17) to first order, is formed by Eq.(6.46) aside from a vector equation: 


$$
\begin{aligned}
& \vec{\nabla} \cdot \vec{\sigma}=\vec{\nabla} \cdot\left(\boldsymbol{\Sigma} \cdot \vec{E}_{1}\right)=0 \\
& \frac{\partial \vec{\sigma}}{\partial t}-\vec{\nabla} \times\left(\boldsymbol{\Omega} \cdot \vec{H}_{1}\right)=\frac{\partial}{\partial t}\left(\boldsymbol{\Sigma} \cdot \vec{E}_{1}\right)-\vec{\nabla} \times\left(\boldsymbol{\Omega} \cdot \vec{H}_{1}\right)=0,
\end{aligned}
$$

where now we have introduced the symmetric tensors

$$
\begin{aligned}
& \boldsymbol{\Sigma}=\frac{\partial \varphi}{\partial X_{0}} \mathbb{I}_{3}+2 \frac{\partial^{2} \varphi}{\partial X_{0}^{2}}\left(\vec{E}_{0} \otimes \vec{E}_{0}\right) \\
& \boldsymbol{\Omega}=\frac{\partial \varphi}{\partial X_{0}} \mathbb{I}_{3}-2 \frac{\partial^{2} \varphi}{\partial Y_{0}^{2}}\left(\vec{E}_{0} \otimes \vec{E}_{0}\right),
\end{aligned}
$$

which will be useful in simplifying the notations in the sequel. The perturbing fields must also satisfy the first set of Maxwell equations. Expanding (5.18) up to the first order we obtain

$$
\begin{aligned}
\vec{\nabla} \times \vec{E}_{1} & =-\frac{\partial \vec{H}_{1}}{\partial t} \\
\vec{\nabla} \cdot \vec{H}_{1} & =0
\end{aligned}
$$

Let us look for solutions of these equations which are products of functions of time and space variables for both electric and magnetic fields, of the form

$$
\begin{aligned}
& \vec{E}_{1}(t, \vec{r})=T_{e}(t) \cdot \vec{e}(\vec{r}) \\
& \vec{H}_{1}(t, \vec{r})=T_{h}(t) \cdot \vec{h}(\vec{r}) .
\end{aligned}
$$

In this way we are lead to the first-order equations for the time variables

$$
\begin{aligned}
& \dot{T}_{e}=\lambda T_{h} \\
& \dot{T}_{h}=-\mu T_{e},
\end{aligned}
$$

where $\lambda$ and $\mu$ are separation constants. By deriving these equations we are lead to the system

$$
\begin{aligned}
& \ddot{T}_{e}(t)+\Gamma T_{e}(t)=0 \\
& \ddot{T}_{h}(t)+\Gamma T_{h}(t)=0,
\end{aligned}
$$

with $\Gamma=\lambda \cdot \mu$. The identification $\lambda=\mu=\sqrt{\Gamma}$ can be introduced without loss of generality, as a consequence of the first-order equations and the positivity of $\Gamma$, which will be established below. The eigenvalue $\Gamma$ being positive, the solutions (normalized to unity) take the form

$$
\begin{aligned}
& T_{e}(t)=\cos (\sqrt{\Gamma} t+\delta) \\
& T_{h}(t)=\sin (\sqrt{\Gamma} t+\delta),
\end{aligned}
$$

where $\delta$ is the same constant phase for both solutions. In this case the eigenfunctions remain bounded as time evolves and the soliton is dynamically stable. The field equations for the spatial components are

$$
\begin{aligned}
\vec{\nabla} \cdot(\boldsymbol{\Sigma} \cdot \vec{e}) & =0 \\
\vec{\nabla} \times(\boldsymbol{\Omega} \cdot \vec{h}) & =\sqrt{\Gamma} \boldsymbol{\Sigma} \cdot \vec{e},
\end{aligned}
$$


where we have used the definitions (6.57). Note that the first of Eqs. (6.63) is an immediate consequence of the second one. Moreover, the first set of Maxwell equations leads to

$$
\begin{aligned}
\vec{\nabla} \times \vec{e} & =\sqrt{\Gamma} \vec{h} \\
\vec{\nabla} \cdot \vec{h} & =0 .
\end{aligned}
$$

We shall now introduce a four-potential $A^{\mu}$ for the perturbing fields, defined in the Hamilton gauge $\left(A^{0}=0\right)$ in such a way that 30

$$
\begin{aligned}
& \vec{E}_{1}=-\frac{\partial \vec{A}}{\partial t} \\
& \vec{H}_{1}=\vec{\nabla} \times \vec{A}
\end{aligned}
$$

This vector potential is determined up to the gradient of an arbitrary time-independent scalar field. In terms of this vector potential, the first set of field equations (6.58) are identically satisfied while the second set (6.56) becomes

$$
\begin{aligned}
& \frac{\partial}{\partial t}(\vec{\nabla} \cdot(\boldsymbol{\Sigma} \cdot \vec{A}))=0 \\
& \frac{\partial^{2}}{\partial t^{2}}(\boldsymbol{\Sigma} \cdot \vec{A})+\vec{\nabla} \times(\boldsymbol{\Omega} \cdot(\vec{\nabla} \times \vec{A}))=0,
\end{aligned}
$$

If we write equations (6.65) for the separated functions (6.59), by integrating in time the first one and using (6.60), we obtain the general form of the vector potential for these functions as

$$
\vec{A}(t, \vec{r})=T_{a}(t) \vec{a}(\vec{r})+\vec{\nabla} \phi(\vec{r}),
$$

where $\phi(\vec{r})$ is a time-independent function and

$$
T_{a}(t)=\frac{T_{h}(t)}{\sqrt{\Gamma}} \quad ; \quad \vec{a}(\vec{r})=\vec{e}(\vec{r}) .
$$

This vector potential, determined in Eq.(6.67) up to the gradient of a time-independent scalar field, becomes univocally fixed by requiring its form to separate in time and space variables, taking the form of the first term in the r.h.s of Eq.(6.67). In terms of this potential, using the definitions (6.57), the field equations for the spatial part of the perturbation reduce to the unique vector equation

$$
\vec{\nabla} \times(\Omega \cdot(\vec{\nabla} \times \vec{a}))=\Gamma \Sigma \cdot \vec{a}
$$

which outlines the eigenvalue problem for the linear oscillations in this electromagnetic case.

As in the scalar case, the standard analysis of this problem can be performed for the ESS solitons of admissible generalized electromagnetic field models without any reference to the explicit form of the lagrangian density. In this way we shall show that two given

\footnotetext{
${ }^{30}$ This gauge-fixing condition is allowed by the gauge invariance of the equations for the perturbing fields (6.56)- (6.58) which are independent from any gauge choice for the unperturbed fields.
} 
eigenfunctions $\vec{a}_{1}(\vec{r})$ and $\vec{a}_{2}(\vec{r})$ associated to the eigenvalues $\Gamma_{1}$ and $\Gamma_{2}$, respectively, are orthogonal and finite-norm with respect to the scalar product

$$
<\vec{a}_{1} \cdot \vec{a}_{2}>=\zeta \int d_{3} \vec{r}\left(\vec{a}_{1} \cdot \Sigma \cdot \vec{a}_{2}\right)
$$

where $\zeta$ is a normalizing factor. Indeed, multiplying Eq.(6.69) (defined for a given eigenfunction $\vec{a}_{1}$ ) by an eigenfunction $\vec{a}_{2}$, integrating in space, using the identity

$$
\vec{a}_{2} \cdot \vec{\nabla} \times\left(\Omega \cdot\left(\vec{\nabla} \times \vec{a}_{1}\right)\right)=\vec{\nabla} \cdot\left[\vec{a}_{2} \times\left(\Omega \cdot\left(\vec{\nabla} \times \vec{a}_{1}\right)\right)\right]+\left(\vec{\nabla} \times \vec{a}_{1}\right) \cdot \Omega \cdot\left(\vec{\nabla} \times \vec{a}_{2}\right),
$$

and taking into account the symmetry of the tensors $\boldsymbol{\Sigma}$ and $\boldsymbol{\Omega}$, we obtain the equation

$$
\begin{aligned}
\int d_{3} \vec{r} \vec{\nabla} \cdot\left[\vec{a}_{2} \times\left(\Omega \cdot\left(\vec{\nabla} \times \vec{a}_{1}\right)\right)\right] & +\int d_{3} \vec{r}\left(\vec{\nabla} \times \vec{a}_{1}\right) \cdot \Omega \cdot\left(\vec{\nabla} \times \vec{a}_{2}\right)= \\
& =\Gamma_{1} \int d_{3} \vec{r}\left(\vec{a}_{1} \cdot \Sigma \cdot \vec{a}_{2}\right) .
\end{aligned}
$$

Owing to the boundary conditions the first integral in the 1.h.s of this equation vanishes while the second one converges. By permuting the indices and subtracting we finally obtain

$$
\left(\Gamma_{1}-\Gamma_{2}\right) \int d_{3} \vec{r}\left(\vec{a}_{1} \cdot \Sigma \cdot \vec{a}_{2}\right)=\left(\Gamma_{1}-\Gamma_{2}\right)<\vec{a}_{1} \cdot \vec{a}_{2}>=0 .
$$

We see that the eigenfunctions associated to different eigenvalues are orthogonal with respect to the scalar product (6.70). Moreover, if $\vec{a}_{1}=\vec{a}_{2}$ we obtain from (6.72)

$$
\Gamma \int d_{3} \vec{r}(\vec{a} \cdot \Sigma \cdot \vec{a})=\int d_{3} \vec{r}(\vec{\nabla} \times \vec{a}) \cdot \Omega \cdot(\vec{\nabla} \times \vec{a}),
$$

and both integrals converge. The integrand in the 1.h.s. of this equation can be written as

$$
(\vec{a} \cdot \Sigma \cdot \vec{a})=\frac{\partial \varphi}{\partial X_{0}}\left((\vec{a})^{2}-\left(\vec{a} \cdot \frac{\vec{r}}{r}\right)^{2}\right)+\left(\frac{\partial \varphi}{\partial X_{0}}+2 \vec{E}_{0}^{2} \frac{\partial^{2} \varphi}{\partial X_{0}^{2}}\right)\left(\vec{a} \cdot \frac{\vec{r}}{r}\right)^{2},
$$

and is positive for ESS soliton solutions of any admissible electromagnetic model. The integrand of the r.h.s. of (6.74) takes the form

$$
\begin{aligned}
(\vec{\nabla} \times \vec{a}) \cdot \Omega \cdot(\vec{\nabla} \times \vec{a}) & =\frac{\partial \varphi}{\partial X_{0}}\left((\vec{\nabla} \times \vec{a})^{2}-\left(\frac{\vec{r}}{r} \cdot \vec{\nabla} \times \vec{a}\right)^{2}\right)+ \\
& +\left(\frac{\partial \varphi}{\partial X_{0}}-2 \vec{E}_{0}^{2} \frac{\partial^{2} \varphi}{\partial Y_{0}^{2}}\right)\left(\frac{\vec{r}}{r} \cdot \vec{\nabla} \times \vec{a}\right)^{2} .
\end{aligned}
$$

This term is positive if (and only if) the condition for static stability (6.54) is fulfilled. Under this condition the eigenvalues $\Gamma$ are well defined and positive and, consequently, the behaviour of any initially bounded perturbation remains bounded as time evolves. We conclude that the statically stable ESS solitons of admissible electromagnetic models which satisfy (6.54) are also dynamically stable. Moreover, the spectrum of eigenvalues is discrete and the eigenfunctions generate the functional space of the physical vector potentials, which can be written as

$$
\vec{A}(t, \vec{r})=\sum_{n} C_{n} \sin \left(\sqrt{\Gamma_{n}} t\right) \vec{a}_{n}(\vec{r})
$$


and are in a one-to-one correspondence with the physical perturbed states of the soliton. Indeed, any charge-preserving perturbation of the soliton field is described, in the Hamilton gauge, by vector potentials which can be obtained from one (and only one) of the form (6.77) by the addition of gradients of time-independent scalar functions.

The analysis of the spatial structure of the eigenfunctions and physical perturbations can now be performed by separating in radial and angular parts the components of the vector potentials $\vec{a}_{n}(\vec{r})$ in the natural basis of the polar coordinate system. This procedure, which is standard in spherically-symmetric physical problems [51, will determine the asymptotic and central-behaviour of the perturbing fields, as in the scalar case already considered. We shall leave this study for future developments.

\subsection{Stability of generalized non-abelian gauge solitons.}

Owing to the essential self-interactions involving the field potentials, the treatment of the static and dynamic stability of the solitons for generalized non-abelian gauge models is more involved than in the abelian case. A detailed study of the stability of some extended static finite-energy solutions for the standard Yang-Mills model has been performed in Ref. [47, where similar difficulties arise. Most methods of that work can be generalized to the present situation and we shall follow this way in analyzing the stability behaviour of the ESS solutions (5.40).

Consider a finite-energy ESS solution of the field equations (5.33) of the form (5.40) and introduce small regular perturbing fields through the definitions

$$
\begin{gathered}
\vec{E}_{a}(r)=-\vec{\nabla} A_{a}^{0}(r), \vec{H}_{a}=0, \vec{A}_{a}=0 \\
\delta \vec{E}_{a}(\vec{r}), \delta \vec{H}_{a}(\vec{r}), \delta A_{a}^{0}(\vec{r}), \delta \vec{A}_{a}(\vec{r}) .
\end{gathered}
$$

To first order these fields are related through

$$
\begin{aligned}
& \delta \vec{E}_{a}(\vec{r})=-\vec{\nabla} \delta A_{a}^{0}-\frac{\partial \delta \vec{A}_{a}}{\partial t}-g \sum_{b c} C_{a b c} \delta \vec{A}_{b} A_{c}^{0} \\
& \delta \vec{H}_{a}(\vec{r})=\vec{\nabla} \times \delta \vec{A}_{a},
\end{aligned}
$$

and they are assumed to leave invariant the color charges $Q_{a}$ associated to the unperturbed solution. To first-order the modifications of the charge densities are obtained from the perturbation of the time-components of the field equations (5.33) (the generalized Gauss laws) and read

$$
\vec{\nabla} \cdot \vec{\sigma}_{a}=-g \sum_{b c} C_{a b c} \frac{\partial \varphi}{\partial X_{0}} \delta \vec{A}_{b} \cdot \vec{E}_{c},
$$

where

$$
\vec{\sigma}_{a}=\frac{\partial \varphi}{\partial X_{0}} \delta \vec{E}_{a}+2 \frac{\partial^{2} \varphi}{\partial X_{0}^{2}}\left(\sum_{p} \vec{E}_{p} \cdot \delta \vec{E}_{p}\right) \vec{E}_{a},
$$

and the modifications of the total charges read 


$$
\Delta Q_{a}=\frac{1}{4 \pi} \int d_{3} \vec{r} \vec{\nabla} \cdot \vec{\sigma}_{a}=-g \sum_{b c} C_{a b c} \int d_{3} \vec{r} \frac{\partial \varphi}{\partial X_{0}} \delta \vec{A}_{b} \cdot \vec{E}_{c}=0 .
$$

The first-order perturbations of the vector equations, given by the spatial components of Eqs.(5.33) (the generalized Ampère laws) read

$$
-\frac{\partial}{\partial t} \vec{\sigma}_{a}+\vec{\nabla} \times \vec{\omega}_{a}=-g \sum_{b c} C_{a b c} \frac{\partial \varphi}{\partial X_{0}}\left[\delta A_{b}^{0} \vec{E}_{c}+A_{b}^{0} \delta \vec{E}_{c}\right],
$$

where

$$
\vec{\omega}_{a}=\frac{\partial \varphi}{\partial X_{0}} \delta \vec{H}_{a}-2 \frac{\partial^{2} \varphi}{\partial Y_{0}^{2}}\left(\sum_{p} \vec{E}_{p} \cdot \delta \vec{E}_{p}\right) \vec{E}_{a} .
$$

The r.h.s. in Eq.(6.80) is the color-charge density carried by the perturbations and its spatial integral must vanish, according to our initial assumptions. This requirement restricts the asymptotic behaviour of $\vec{\sigma}_{a}$ and, as for the other field models already considered, leads to boundary conditions to be satisfied by the perturbing fields. Moreover, similarly to the multicomponent scalar case, the color charges of the unperturbed solution $\left(Q_{a}\right)$ fix a direction in the color-charge space (called in Ref.[47] "electromagnetic" direction, while the orthogonal directions are termed "charged"). Owing to the first-integral equation (5.38), the potentials $A_{a}^{0}$ and the fields $\vec{E}_{a}$ of the ESS solutions lie in this direction. For the perturbing fields to remain purely electromagnetic the associated charge densities that are induced by them must vanish and, owing to Eq.(6.80), $\delta \overrightarrow{A_{b}}$ must also lie in this direction. In what follows, we shall prove the stability of the finite-energy ESS solutions against this kind of non-charged perturbations.

Now let us analyze the variations of the energy functional. The first variation is obtained by perturbing the spatial integral of (5.35) (this is a gauge-invariant quantity, as well as its variations) around the ESS solutions, which reads

$$
\Delta_{1} \epsilon=-2 \int d_{3} \vec{r} \sum_{a} \vec{\nabla} \cdot\left[A_{a}^{0} \vec{\sigma}_{a}\right]+2 \int d_{3} \vec{r} \sum_{a} A_{a}^{0} \vec{\nabla} \cdot \vec{\sigma}_{a} .
$$

The first integral in this expression vanishes, owing to the boundary conditions. Using Eq.(6.80) the variation becomes

$$
\Delta_{1} \epsilon=-2 g \int d_{3} \vec{r} \sum_{a b c} C_{a b c} \frac{\partial \varphi}{\partial X_{0}} A_{a}^{0} \delta \vec{A}_{b} \cdot \vec{E}_{c} .
$$

As expected this expression vanishes, owing to the parallelism of $A_{a}^{0}$ and $\vec{E}_{c}$ in the color space (see Eqs.(5.40) and (5.43)) and the antisymmetry of the structure constants.

In obtaining the second variation of the energy functional we follow the same steps as in the abelian case. First we expand the generalized Gauss law to the second order. After the cancellation of the first-order terms we are lead to

$$
\vec{\nabla} \cdot \vec{\omega}_{a}=-g \sum_{b c} C_{a b c}\left[\frac{\partial \varphi}{\partial X_{0}} \delta \vec{A}_{b} \cdot \delta \vec{E}_{c}+2 \frac{\partial^{2} \varphi}{\partial X_{0}^{2}}\left(\sum_{p} \vec{E}_{p} \cdot \delta \vec{E}_{p}\right) \delta \vec{A}_{b} \cdot \vec{E}_{c}\right],
$$

where now 


$$
\begin{aligned}
\vec{\omega}_{a} & =2 \frac{\partial^{2} \varphi}{\partial X_{0}^{2}}\left(\sum_{p} \vec{E}_{p} \cdot \delta \vec{E}_{p}\right) \delta \vec{E}_{a}+\frac{\partial^{2} \varphi}{\partial X_{0}^{2}} \sum_{p}\left(\delta \vec{E}_{p}^{2}-\delta \vec{H}_{p}^{2}\right) \vec{E}_{a}+ \\
& +2 \frac{\partial^{3} \varphi}{\partial X_{0}^{3}}\left(\sum_{p} \vec{E}_{p} \cdot \delta \vec{E}_{p}\right)^{2} \vec{E}_{a}+2 \frac{\partial^{3} \varphi}{\partial X_{0} \partial Y_{0}^{2}}\left(\sum_{p} \vec{E}_{p} \cdot \delta \vec{H}_{p}\right)^{2} \vec{E}_{a}+ \\
& +2 \frac{\partial^{2} \varphi}{\partial Y_{0}^{2}}\left(\sum_{p} \vec{E}_{p} \cdot \delta \vec{H}_{p}\right) \delta \vec{H}_{a} .
\end{aligned}
$$

By expanding the integral of (5.35) up to second order and using Eqs.(6.80) and (6.87) the second variation of the energy becomes

$$
\begin{aligned}
\Delta_{2} \epsilon & =\int d_{3} \vec{r}\left[\frac{\partial \varphi}{\partial X_{0}} \sum_{a} \delta \vec{E}_{a}^{2}+2 \frac{\partial^{2} \varphi}{\partial X_{0}^{2}}\left(\sum_{a} \vec{E}_{a} \cdot \delta \vec{E}_{a}\right)^{2}\right]+ \\
& +\int d_{3} \vec{r}\left[\frac{\partial \varphi}{\partial X_{0}} \sum_{a} \delta \vec{H}_{a}^{2}-2 \frac{\partial^{2} \varphi}{\partial Y_{0}^{2}}\left(\sum_{a} \vec{E}_{a} \cdot \delta \vec{H}_{a}\right)^{2}\right]- \\
& -2 \int d^{3} \vec{r} \vec{\nabla} \cdot\left(A_{0}^{a} \cdot \vec{\omega}^{a}\right)-2 g \int d_{3} \vec{r} \frac{\partial \varphi}{\partial X_{0}} \sum_{a b c} C_{a b c} A_{a}^{0} \delta \vec{A}_{b} \cdot \delta \vec{E}_{c} .
\end{aligned}
$$

Once again, the divergence term in this expression vanishes owing to the boundary conditions. The integrand of the last term is the scalar product in color space between the potential $A_{a}^{0}$ of the unperturbed field and the first component of the second-order perturbation of the color-charge density in the r.h.s of Eq.(6.87). For electromagnetic perturbations this component must satisfy the condition

$$
\frac{\partial \varphi}{\partial X_{0}} \sum_{a b c} C_{a b c} A_{a}^{0} \delta \vec{A}_{b} \cdot \delta \vec{E}_{c}=0,
$$

(note that the remaining component in the r.h.s. of Eq.(6.87) lies already in the electromagnetic direction). Consequently, the last term in Eq.(6.89) must vanish and the second variation of the energy takes a form similar to that of the abelian case. We can now determine the conditions for stability of the finite-energy ESS solutions in this non-abelian case through a similar argumentation. As easily seen stability requires the lagrangian-density function $\varphi(X, Y)$ to satisfy the condition

$$
\frac{\partial \varphi}{\partial X} \geq 2 X \frac{\partial^{2} \varphi}{\partial Y^{2}}
$$

in all the range of values of the gauge invariants $(X, Y=0)$ defined by the solution. This condition is formally the same as in the abelian case and is also necessary and sufficient for the stability of the solitons against electromagnetic perturbations. Obviously, it is a gauge-invariant criterion.

The analysis of the dynamical stability of non-abelian solitons should now be performed starting with Eqs.(6.80) and (6.83) and following similar steps as in the abelian case. But the presence of the antisymmetric structure constants and the symplectic character of the eigenvalue problem require new qualitative procedures and longer calculations which would lengthen excessively the contents of this work. This issue will be approached elsewhere. 


\section{Conclusions and perspectives}

In this work we have solved the problem of characterizing a large class of physically consistent relativistic lagrangian field theories in three-space dimensions, supporting static spherically symmetric non-topological soliton solutions. The fields concerned were one and many-components scalar fields (whose lagrangian densities depend on the kinetic term alone) and generalized gauge fields of compact semi-simple Lie groups. This characterization is exhaustive and leads to the classification of such models into six types, according to the central and asymptotic behaviours of the soliton fields. We have performed a broad analysis of the linear stability of the solutions, obtaining necessary and sufficient stability conditions which go beyond the usual Derrick criterion. We also have carried out a general spectral analysis of the linear perturbations around the soliton solutions, confirming their dynamical stability and setting grounds for their quantum extensions.

All these results allow the explicit determination of a large number of examples of such a class of lagrangians, providing a wide panoply of tools for the analysis of diverse physical problems, as those mentioned in the introduction and others. Among these problems let us outline three of particular interest, which we are addressing from the methods developed here.

1) As already discussed in section 3.1., the photon-photon interaction mediated by the QED vacuum can be classically described in terms of effective lagrangians which are polynomial expressions in the gauge invariants $X$ and $Y$ and can be obtained in a perturbative procedure [31], where the lowest order is the well-known Euler-Heisenberg lagrangian [32]

$$
\varphi(X, Y) \sim \eta X+\xi\left(4 X^{2}+7 Y^{2}\right)
$$

( $\eta$ and $\xi$ being positive constants). The sequence of these lagrangians exhibits finite-energy ESS solutions and suggests an interpretation in terms of the screening effects of the vacuum on the field of point-like charges. Unfortunately, as already mentioned, the perturbative expansion involved in this procedure is a low-energy (or a low-intensity field) approximation and is not accurate to describe the strong fields present near the center of the ESS solutions. It is thus necessary to explore this issue with other effective lagrangians, obtained from the perturbative renormalization of the self-energy of point-like fields. The analysis of this problem is in progress [41], 552.

2) Scalar field models as that of the example treated in section 3.4., which belong to the case B-3, exhibiting short-ranged SSS solutions (solitons or not), can be extended to generalized gauge field models supporting similar ESS solutions. This behaviour, which is related to the form of the lagrangian density around the vacuum, may arise in effective actions for some fundamental forces. If we assume the effective dynamics of the non-abelian gauge fields in electroweak interactions to be described by this kind of lagrangians, the short range of these forces could be explained in terms of the non-linear self-couplings among these fields, coming from the integration of some higher energy degrees of freedom of a more fundamental theory. In this case the appeal to any symmetry breaking or Higgs mechanism should become superfluous. In our sense, this alternative deserves to be thoroughly explored.

3) A new approach to the phenomenological description of the hadronic structure can be envisaged, using the results of section 6 on the spectral analysis of the excitations of the 
(multi-) scalar or generalized gauge-invariant solitons. In the phenomenological descriptions based on the Skyrme model the hadron arises as a topological soliton of a non-linear field theory. Other models, which are believed to give an effective low-energy approach to the non-perturbative regime of QCD (as the Friedberg-Lee model [7], [8] and related theories), describe hadrons as confined states of quarks in non-topological solitonic bags of non-linear phenomenological fields. As an alternative to these viewpoints, we are considering a generalized gauge-invariant lagrangian model for gauge fields ("gluons") coupled to a quark-like fermionic sector and implementing properly chosen symmetries. Such a phenomenological model may be interpreted as an effective lagrangian for QCD or, alternatively, as a fieldtheoretical low-energy limit of string theory. The classical generalized gauge-field lagrangian can exhibit soliton solutions in absence of other fields. If such solutions are minima of the functional of energy of the full action, their small perturbations will involve fermionic and bosonic modes. The quantization of these modes leads to "quasi-quarks" and "quasi-gluons" as quantum excitations of the soliton field. This quantum extension becomes a model for the hadron containing these particles. In this picture the confinement would be a consequence of the fact that quarks are quasi-particles associated to these quantum excitations and (as the phonons in a solid) they cannot exist outside the hadron.

Another domain where the results of this work could be useful concerns the search for self-gravitating (scalar and gauge) field configurations in General Relativity [23]. The classification of the lagrangian field theories considered here, supporting non-topological solitons in flat space, can be extended to the static spherically symmetric solutions of the Einstein equations resulting from the coupling of these fields to gravitation. Indeed, we have verified that these equations have first-integrals which have the same form as (or can be closely related to) the ones obtained from the corresponding field theories in flat space (of the generic form of Eqs.(2.4) or (5.19) ). This result opens the possibility of generalizing to the gravitational case many of the methods and results obtained here [53]. We will continue to address this topic in future work.

It would be also interesting to study the soliton solutions of generalized non-abelian gauge field theories with other ansatzes than the ESS one. In fact, such a kind of solutions have been already found for the $\mathrm{SU}(2)$ non-abelian B-I theory within the "monopole ansatz" [15]. This issue should be a theme for a future investigation.

Let us conclude with some comments concerning an important question which has not been considered here. It refers to the analysis of propagation of wave-like solutions of these models. As can be easily seen, all these theories exhibit plane wave solutions propagating with the speed of light. But, owing to the non-linear self-coupling, they also support other radiative solutions propagating with more complex dispersion relations. In most cases such waves evolve towards spatially-singular configurations. Roughly speaking, the wave fronts travelling with velocities which are dependent on the values of the fields at every point tend to cumulate, generating discontinuities and shocks after a critical time. Regularly evolving wave solutions of a system of field equations are called exceptional. If all the wave solutions of a given system are exceptional, the system is called completely exceptional [54]. A detailed analysis of the problem of wave propagation for generalized electromagnetic field models was performed by G. Boillat [55], who established the complete exceptionality of the Born-Infeld electrodynamics. Moreover, B-I is the only admissible generalized electro- 
magnetic field theory (with asymptotically coulombian elementary solutions) exhibiting this property. Nevertheless, the Boillat analysis considers only models which satisfy the condition $\frac{\partial \varphi}{\partial X}(X=0, Y=0)=1$ (case B-2) and, consequently, excludes the models belonging to B-1 and B-3 cases. It would be interesting to extend the Boillat analysis for these cases. However, when one considers the extensions of B-I electrodynamics to the non-abelian case or in the Kaluza-Klein context, this exceptionality character is lost [56].

\section{Acknowledgments}

Many parts of this work have been discussed with several colleagues. We are indebted to Drs. E. Alvarez, L. Bel, S. Bonazzola, B. Carter, M.A. Cobas, B. Coll, R. Hakim, J. -L. Jaramillo, J. Larena, T. Lehner, Y. Lozano, J. Madore, L. Mornas, A. Nieto, M.A.R. Osorio, A.R. Plastino, J.A. Rodriguez-Mendez and V. Vento for useful discussions and suggestions.

\section{References}

[1] G. Mie, Annalen der Physik 37 (1912) 551.

[2] M. Born, Proc. R. Soc. London A 143 (1934) 410.

[3] M. Born, L. Infeld, Proc. R. Soc. London A 144 (1934) 425.

[4] A. C. Scott, F. Y. Chu, D. W. McLaughlin, Proc. IEEE 61 (1973) 1473.

[5] G. 't Hooft, Nucl. Phys. B 79 (1974) 276; A. M. Polyakov, JETP Lett. 20 (1974) 194.

[6] T. H. R. Skyrme, Proc. R. Soc. London A 260 (1961) 127;

T. H. R. Skyrme, Nucl. Phys. 31 (1962) 556;

E. Witten, Nucl. Phys. B 223 (1983) 422.

[7] R. Friedberg, T. D. Lee, Phys. Rev. D 15 (1977) 1694;

R. Friedberg, T. D. Lee, Phys. Rev. D 16 (1977) 1096;

R. Friedberg, T. D. Lee, Phys. Rev. D 18 (1978) 2623.

[8] T. D. Lee, Y. Pang, Phys. Rep. 221 (1992) 251.

[9] S. Deser, M. J. Duff, C. J. Isham, Nucl. Phys. B 114 (1976) 29.

[10] G. H. Derrick, J. Math. Phys. 5 (1964) 1252.

[11] D. A. Nicol, J. Phys. G 4 (1978) 1363;

C. Adam, J. Sanchez-Guillen, R. A. Vazquez, A. Wereszczynski, J. Math. Phys. 47 (2006) 052302.

[12] H. Aratyn, L. A. Ferreira, A. Zimerman, Phys. Lett. B 456 (1999) 162;

H. Aratyn, L. A. Ferreira, A. Zimerman, Phys. Rev. Lett. 83 (1999) 1723.

[13] L. Faddeev, A. Niemi, Nature 387 (1997) 58;

L. Faddeev, A. Niemi, Phys. Rev. Lett. 82 (1999) 1624;

E. Langmann, A. Niemi, Phys. Lett. B 463 (1999) 252.

[14] H. -J. Lee, B. -Y. Park, D. -P. Min, M. Rho, V. Vento, Nucl. Phys. A 723 (2003) 427;

H. -J. Lee, B. -Y. Park, M. Rho, V. Vento, Nucl. Phys. A 726 (2003) 69;

B. -Y. Park, M. Rho, V. Vento, Nucl. Phys. A 736 (2004) 129.

[15] D. V. Gal'tsov, R. Kerner, Phys. Rev. Lett. 84 (2000) 5955.

[16] E. S. Fradkin, A. A. Tseytlin, Phys. Lett. B 163 (1985) 123;

R. G. Leigh, Mod. Phys. Lett. A 4 (1989) 2767;

E. Witten, Nucl. Phys. B 460 (1996) 335;

A. A. Tseytlin, Nucl. Phys. B 501 (1997) 41;

M. Perry, J. H. Schwarz, Nucl. Phys. B 489 (1997) 47.

[17] O. V. Pavlovsky, Phys. Lett. B 538 (2002) 202.

[18] G. W. Gibbons, K. Hashimoto, JHEP 0009 (2000) 013.

[19] T. Ortin, "Gravity and strings", Cambridge Monographs on Mathematical Physics, C.U.P., 2004.

[20] S. Deser, Phys. Lett. B 64 (1976) 463;

S. Coleman, Comm. Math. Phys. 55 (1977) 113.

[21] R. Bartnik, J. McKinnon, Phys. Rev. Lett. 61 (1988) 141. 
[22] E. Ayón-Beato, A. García, Phys. Rev. Lett. 80 (1998) 5056;

E. Ayón-Beato, A. García, Gen. Rel. Grav. 31 (1999) 629.

[23] M. Demianski, Found. of Phys. 16 (1986) 187;

H. P. de Oliveira, Class. Quant. Grav. 11 (1994) 1469;

M. S. Volkov, D. V. Gal'tsov, Phys. Rept. 319 (1999) 1;

V. V. Dyadichev, D. V. Gal'tsov, Phys. Lett. B 486 (2000) 431.

[24] T. D. Lee, Phys. Rev. D 35 (1987) 3637.

[25] S. Coleman, Nucl. Phys. B 262 (1985) 263;

K. Enqvist, A. Mazumdar, Phys. Rept. 380 (2003) 99.

[26] A. Kusenko, P. Steinhardt, Phys. Rev. Lett. 87 (2001) 141301.

[27] J. A. Frieman, G. B. Gelmini, M. Gleiser, E. Kiolb, Phys. Rev. Lett. 60 (1988) 2101.

[28] V. V. Dyadichev, D. V. Gal'tsov, A. G. Zorin, M. Y. Zotov, Phys. Rev. D 65 (2002) 084007;

A. Füzfa, J. -M. Alimi, Phys. Rev. Lett. 97 (2006) 061301;

A. Füzfa, J. -M. Alimi, Phys. Rev. D 73 (2006) 023520.

[29] C. Armendariz-Picon, T. Damour, V. F. Mukhanov, Phys. Lett. B 458 (1999) 209.

[30] C. Armendariz-Picon, V. F. Mukhanov, P. J. Steinhardt, Phys. Rev. D 63 (2001) 103510;

C. Armendariz-Picon, E. A. Lim, JCAP 0508 (2005) 007.

[31] A. Dobado, A. Gḿez-Nicola, A. L. Maroto, J. R. Peláez, "Effective Lagrangians for the Standard Model", Springer-Verlag, Berlin, Heidelberg, 1997.

[32] W. Heisenberg, H. Euler, Z. Phys. 98 (1936) 714; J. Schwinger, Phys. Rev. 82 (1951) 664.

[33] Z. Bialynicka-Birula, I. Bialynicki-Birula, Phys. Rev. D 2 (1970) 2341.

[34] J. Diaz-Alonso, Phys. Rev. D 28 (1983) 791.

[35] J. Diaz-Alonso, D. Rubiera-Garcia, Phys. Lett. B 653 (2007) 445.

[36] J. Diaz-Alonso, D. Rubiera-Garcia, Phys. Lett. B 657 (2007) 257.

[37] J. L. Anderson, "Principles of Relativity Physics", Academic Press, 1967.

[38] P. Ramond, "Field theory. A modern primer", The Benjamin, Cummings Publishing Company, 1981.

[39] T. D. Lee, "Particle Physics and Introduction to Field Theory", Harwood Academic Publishers, GmbH, 1981.

[40] L. Faddeev, Harvard-Princeton lectures, IAS preprint, 1975.

[41] J. Diaz-Alonso, D. Rubiera-Garcia, "Vacuum polarization and solitons in Quantum Electrodynamics", Contribution to GraviMAS FEST. Palma de Mallorca, Spain. February 11-13 (2008).

[42] L. D. Landau, E. M. Lifshitz, "The Classical Thory of Fields", Pergamon, Oxford, 1975.

[43] S. Coleman, L. Smarr, Comm. Math. Phys. 56 (1977) 1.

[44] A. Abouelsaood, C. G. Callan, C. R. Nappi, S. A. Yost, Nucl. Phys. B 280 (1987) 599;

K. Shiraishi, S. Hirenzaki, Int. Jour. of Mod. Phys. A 6 (1991) 2635;

D. Brecher, Phys. Lett. B 442 (1998) 117;

E. Serie, T. Masson, R. Kerner, Phys. Rev. D 68 (2003) 125003.

[45] N. J. Zabuski, M. D. Kruskal, Phys. Rev. Lett. 15 (1965) 240.

[46] A. A. Chernitsky, Helv. Phys. Acta. 71 (1998) 274.

[47] R. Jackiw, P. Rossi, Phys. Rev. D 21 (1980) 426.

[48] R. Courant, D. Hilbert, "Methods of Mathematical Physics", Vol.1, Chapter 6, Section 3, Wiley, 1989.

[49] G. N. Watson, "A treatise of the theory of Bessel functions", Cambridge Univ. Press, Cambridge, 1944.

[50] A. Sommerfeld, "Partial differential equations in Physics", Academic Press, London, 1964.

[51] S. Banazzola, J. A. Marck, J. Comput. Phys. 87 (1990) 201; S. Bonazzola, L. Villain, M. Bejger, Class. Quant. Grav. 24 (2007) S221.

[52] J. Diaz-Alonso, D. Rubiera-Garcia, in preparation.

[53] J. Diaz-Alonso, D. Rubiera-Garcia, "Soliton solutions in relativistic field theories and gravitation". Proc. of the ERE07, p.193 (2007). arXiv:0712.1702 [hep-th].

[54] P. D. Lax, Commun. Pure Appl. Math. 10 (1957) 537.

[55] G. Boillat, J. Math. Phys. 11 (1970) 941;

G. Boillat, J. Math. Phys. 11 (1970) 1482.

[56] J. P. S. Lemos, R. Kerner, Grav. and Cosmology 6 (2000) 49;

G. W. Gibbons, C. A. R. Herdeiro, Phys. Rev. D 63 (2001) 064006. 